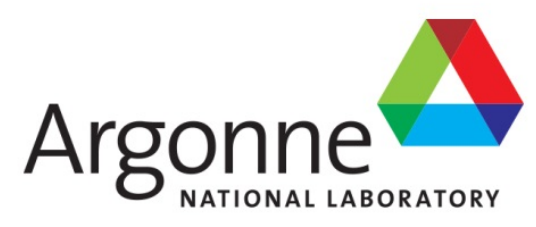

\title{
Optimization of the Dissolution of Molybdenum Disks: FY-16 Results
}

Nuclear Engineering Division 


\begin{abstract}
About Argonne National Laboratory
Argonne is a U.S. Department of Energy laboratory managed by UChicago Argonne, LLC under contract DE-AC02-06 CH11357. The Laboratory's main facility is outside Chicago, at 9700 South Cass Avenue, Argonne, Illinois 60439. For information about Argonne and its pioneering science and technology programs, see www.anl.gov.
\end{abstract}

\title{
DOCUMENT AVAILABILITY
}

Online Access: U.S. Department of Energy (DOE) reports produced after 1991 and a growing number of pre-1991 documents are available free via DOE's SciTech Connect (http://Www.osti.gov/scitech/)

Reports not in digital format may be purchased by the public from the National Technical Information Service (NTS):

U.S. Department of Commerce

National Technical Information Service

5301 Shawnee Rd

Alexandria, VA 22312

unw.ntis.gov

Phone: (800) 553-NTIS (6847) or (703)

605-6000 Fax: (703) 605-6900

Email: orders@ntis.gov

Reports not in digital format are available to DOE and DOE contractors from the Office of Scientific and Technical Information (OST):

U.S. Department of Energy

Office of Scientific and Technical Information

P.O. Box 62

Oak Ridge, TN 37831-0062

unw.osti.gov

Phone: (865) 576-8401

Fax: (865) 576-5728

Email: reports@osti.gov

\section{Disclaimer}

This report was prepared as an account of work sponsored by an agency of the United States Government. Neither the United States Government nor any agency thereof, nor UChicago Argonne, LLC, nor any of their employees or officers, makes any warranty, express or implied, or assumes any legal liability or responsibility for the accuracy, completeness, or usefulness of any information, apparatus, product, or process disclosed, or represents that its use would not infringe privately owned rights. Reference herein to any specific commercial product, process, or service by trade name, trademark, manufacturer, or otherwise, does not necessarily constitute or imply its endorsement, recommendation, or favoring by the United States Government or any agency thereof. The views and opinions of document authors expressed herein do not necessarily state or reflect those of the United States Government or any agency thereof, Argonne National Laboratory, or UChicago Argonne, LLC. 


\section{Optimization of the Dissolution of Molybdenum Disks: FY-16 Results}

by

Peter Tkac, David A. Rotsch, Sergey D. Chemerisov, James L. Bailey, John F. Krebs, and George F. Vandegrift

Nuclear Engineering Division, Argonne National Laboratory

September 2016 



\section{CONTENTS}

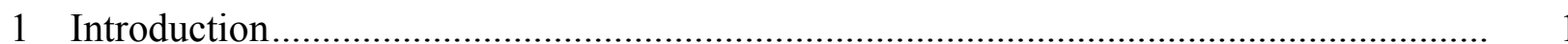

2 Dissolution of Mo Disks to Optimize Production of Mo Targets....................................... 2

3 Optimization of Large-Scale Dissolution of Sintered Mo Disks ........................................ 11

4 Adaptation of Benchtop Optimized Dissolution System to Hot Cell Operations............... 19

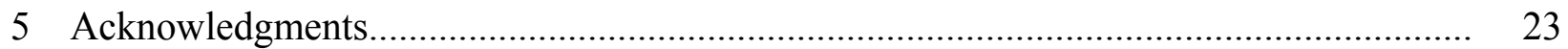

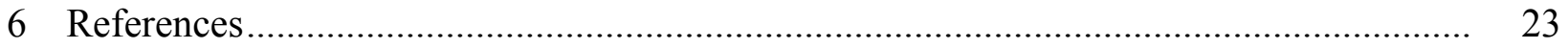

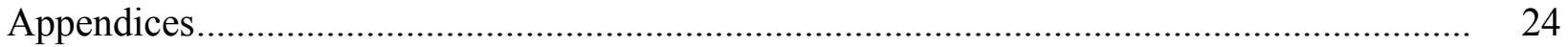

\section{FIGURES}

1 Photographs of $12 \times 1 \mathrm{~mm}$ sintered and 12.4×1.5-1.9 mm 3D-printed Mo disks.......... 2

2 Photographs of $\sim 29.5 \times 2 \mathrm{~mm}$ sintered Mo disks........................................................ 3

3 Photograph of residual small particles that form during the dissolution of lasermelt 3D-printed Mo disks ........................................................................................ 6

$4 \quad$ Floating $29 \times 0.5 \mathrm{~mm}$ sintered Mo disks during dissolution in $40 \mathrm{~mL}$ of $50 \% \mathrm{H}_{2} \mathrm{O}_{2}$; Formation of froth in $2 \mathrm{~L}$ reaction vessel during the dissolution of $12 \mathrm{~g}$ of

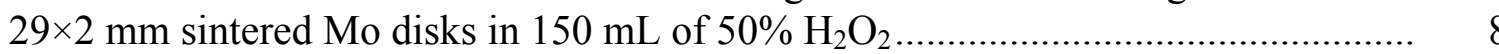

$5 \quad$ Photo of $600 \mathrm{~g}$ of sintered Mo disks placed inside $4 \mathrm{~L}$ dissolution vessel ................... 11

6 Dissolution apparatus for processing of up to $600 \mathrm{~g}$ of sintered Mo disks using $\mathrm{H}_{2} \mathrm{O}_{2}$.

$7 \quad$ Schematic of dissolution setup with placement of thermocouples to monitor temperatures during dissolution of $600 \mathrm{~g}$ batch of sintered Mo disks

8 Temperature profiles during first $15 \mathrm{~min}$ for dissolution of $600 \mathrm{~g}$ of sintered Mo disks in $50 \% \mathrm{H}_{2} \mathrm{O}_{2}$

9 Thermal images of condensers before dissolution started; dissolution vessel during dissolution; flexible adapters between dissolution vessel and condensers; condensers during dissolution; bottom of dissolution vessel during dissolution; condensation flask containing condensed water from dissolution. 
10 Hot cell mockup with view from operator standpoint. No leaded glass is in place......

11 Evaporation vessel with heating mantel on $10 \mathrm{~L}$ reaction vessel; evaporation vessel head with associated stirrer and connections to condenser system; and evaporation condenser system.

12 Dissolution vessel and dissolution vessel condenser system.

A.1 Custom-made dissolution vessel.

\section{TABLES}

1 Characteristics and dissolution results for sintered Mo disks in $40 \mathrm{~mL}$ of $50 \% \mathrm{H}_{2} \mathrm{O}_{2}$.

2 Characteristics and dissolution results for sintered Mo disks from recycled material in $40 \mathrm{~mL}$ of $50 \% \mathrm{H}_{2} \mathrm{O}_{2}$

3 Characteristics and dissolution results for laser-melt 3D-printed Mo disks in $40 \mathrm{~mL}$ of $50 \% \mathrm{H}_{2} \mathrm{O}_{2}$

4 Characteristics and dissolution results for sintered Mo disks in $150 \mathrm{~mL}$ of $50 \%$ $\mathrm{H}_{2} \mathrm{O}_{2}$

5 Characteristics and dissolution results for sintered Mo disks in $50 \% \mathrm{H}_{2} \mathrm{O}_{2}$ preheated to $70^{\circ} \mathrm{C}$

6 Results from optimization of large-scale dissolution experiments 


\section{OPTIMIZATION OF THE DISSOLUTION OF MOLYBDENUM DISKS: FY-16 RESULTS}

\section{INTRODUCTION}

Argonne National Laboratory, with support from the National Nuclear Security Administration's Material Management and Minimization program, is providing technical development assistance to NorthStar Medical Technologies LLC in its pursuit of two pathways for production of molybdenum-99. As a short-term answer to demands, NorthStar plans to use the ${ }^{98} \mathrm{Mo}(\mathrm{n}, \gamma)^{99}$ Mo reaction (neutron capture) pathway at the University of Missouri Research Reactor. As a long-term solution, NorthStar will produce ${ }^{99}$ Mo via the photonuclear reaction, ${ }^{100} \mathrm{Mo}(\gamma, \mathrm{n}){ }^{99} \mathrm{Mo}$, from irradiation of enriched Mo targets by an electron accelerator. Processing of irradiated targets, from either production mode, requires dissolution of the target material in $\mathrm{H}_{2} \mathrm{O}_{2}$ followed by a concentration step, addition of ferric ion to precipitate impurities, and conversion of the final solution to $5 \mathrm{M}$ potassium hydroxide solution of potassium molybdate.

Currently, NorthStar is using pressed and sintered Mo disks as targets. Several options are being considered for the design of Mo targets for the production of ${ }^{99}$ Mo using the $(\gamma, \mathrm{n})$ reaction. In the current design, the target holder contains a series of sintered Mo disks lined up perpendicular to two incident electron beams, one entering from each side of the target stack. In this configuration, the front-most disks absorb most of the heat from the electron beam and need to be thinner to allow for better cooling, while the middle of the target can be thicker. Distribution of the total mass of Mo allows for larger masses of Mo material and thus larger production batches of ${ }^{99} \mathrm{Mo}$.

A limitation of the sintering approach is the production of very thin disks. On the other hand, recent advances in 3D printing allow for laser-melting on metals with high melting points, such as Mo. This approach gives more flexibility in the target design, and much thinner target components can be achieved than when the traditional press-and-sinter approach is used. Another advantage is that an entire target, including the housing, can be fabricated from Mo.

Recent progress on dissolution of sintered Mo disks is well documented [1-4]. We have demonstrated that several factors can play important roles in dissolution behavior: particle size of Mo metal used for production of targets, sintering conditions, degree of open porosity, and thickness of the sintered Mo targets.

Here we report experimental results from studies of small-scale dissolution of sintered Mo disks fabricated from various recycled and commercial Mo materials, and dissolution of 3D-printed Mo disks that were fabricated by Oak Ridge National Laboratory (ORNL). We also report on large-scale dissolution studies with $600 \mathrm{~g}$ batches of sintered Mo disks. 


\section{DISSOLUTION OF MO DISKS TO OPTIMIZE PRODUCTION OF MO TARGETS}

\section{Experimental}

Sintered Mo disks and 3D-printed disks (laser-melt produced) were fabricated by ORNL. Small disks $\left(<2 \mathrm{~g}\right.$ ) were dissolved in $40 \mathrm{~mL}$ of $50 \% \mathrm{H}_{2} \mathrm{O}_{2}$ (Aqua Solutions $\mathrm{H} 3050-4 \mathrm{~L}$ ) preheated to $70^{\circ} \mathrm{C}$ (unless stated otherwise) in a $250 \mathrm{~mL}$ beaker. Larger disks were dissolved in a 2

$\mathrm{L}$ reaction vessel. Photographs of sintered and 3D-printed disks are shown on Figures 1 and 2.

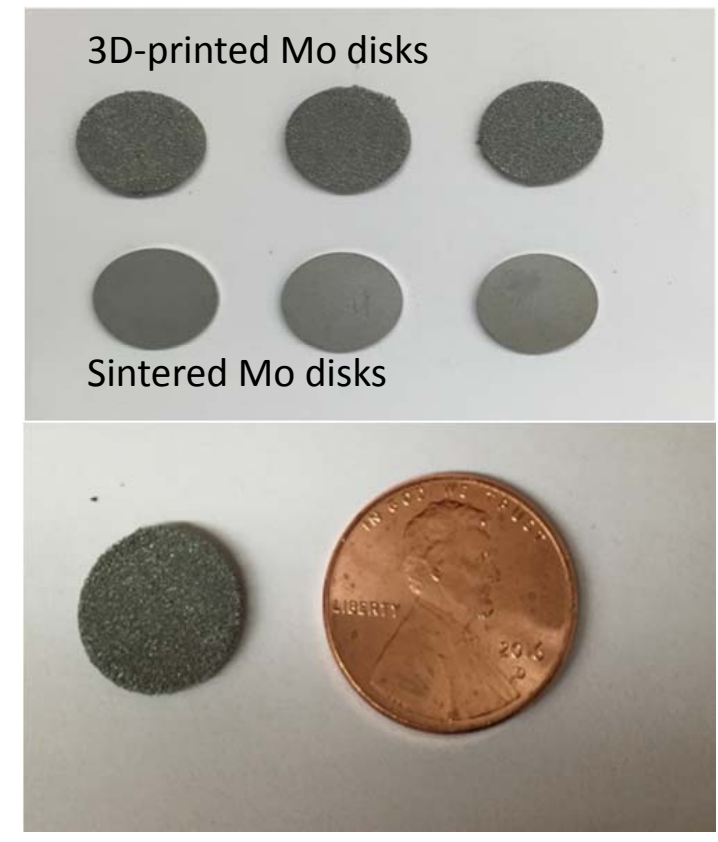

FIGURE 1 Photographs of $12 \times 1 \mathrm{~mm}$ sintered and 12.4×1.5-1.9 mm 3D-printed Mo disks. U.S. penny shown for scale. 


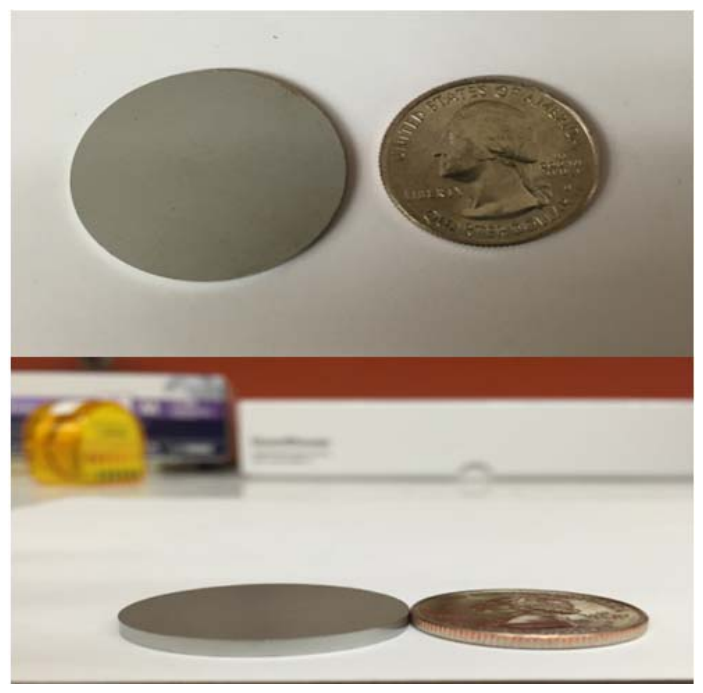

FIGURE 2 Photographs of $\sim 29.5 \times 2 \mathrm{~mm}$ sintered Mo disks. U.S. quarter shown for scale.

\section{Results and Discussion}

\section{$12 \times 1 \mathrm{~mm}$ Sintered Mo Disks}

Sintered Mo disks dissolved quickly with $\mathrm{H}_{2} \mathrm{O}_{2}$ that was preheated to $70^{\circ} \mathrm{C}$. Dissolution of disks from the same batch in room-temperature $\left(24^{\circ} \mathrm{C}\right) \mathrm{H}_{2} \mathrm{O}_{2}$ required an activation period of 3-5 min (the time required to produce enough heat for disks to start dissolving vigorously). Characteristics of sintered Mo disks and dissolution times are listed in Table 1. Dissolution rates were in the range of $\sim 0.76-1.05 \mathrm{~g} / \mathrm{min}$.

TABLE 1 Characteristics and dissolution results for sintered Mo disks in $40 \mathrm{~mL}$ of $50 \% \mathrm{H}_{2} \mathrm{O}_{2}$

\begin{tabular}{|c|c|c|c|c|c|c|c|c|}
\hline Disk I.D. & $\begin{array}{c}\text { Mass } \\
(\mathrm{g})\end{array}$ & $\begin{array}{c}\text { Theor. } \\
\text { Density } \\
(\%)\end{array}$ & $\begin{array}{c}\text { Open } \\
\text { Porosity } \\
(\%)\end{array}$ & $\begin{array}{c}\text { Diameter } \\
(\mathrm{mm})\end{array}$ & $\begin{array}{l}\text { Thickness } \\
(\mathrm{mm})\end{array}$ & $\begin{array}{c}50 \% \mathrm{H}_{2} \mathrm{O}_{2} \\
\text { Temperature } \\
\left({ }^{\circ} \mathrm{C}\right) \\
\end{array}$ & $\begin{array}{l}\text { Diss. } \\
\text { Time } \\
(\mathrm{min}) \\
\end{array}$ & $\begin{array}{c}\text { Diss. } \\
\text { Rate } \\
\text { (g/min) }\end{array}$ \\
\hline S50-12A-5 & 0.986 & 87.9 & 7.9 & 11.99 & 0.94 & 70 & 1.25 & 0.79 \\
\hline S50-12A-7 & 1.008 & 89.1 & 2.7 & 12.01 & 0.94 & 70 & 1.33 & 0.76 \\
\hline S50-12A-8 & 0.994 & 87.9 & 7.8 & 12.01 & 0.97 & 24 & 6.25 & $0.16^{*}$ \\
\hline S50-12A-10 & 0.994 & 86.7 & 8.3 & 12.01 & 0.97 & 70 & 1.00 & 0.99 \\
\hline S50-12B-2 & 1.052 & 87.4 & 8.6 & 11.99 & 1.05 & 70 & 1.00 & 1.05 \\
\hline S50-12B-4 & 1.048 & 87.3 & 8.3 & 11.99 & 1.06 & 24 & 4.00 & $0.26^{+}$ \\
\hline S50-12B-21 & 1.055 & 87.1 & 8.0 & 11.99 & 1.05 & 70 & 1.33 & 0.79 \\
\hline
\end{tabular}

* After an activation period $(5 \mathrm{~min})$, dissolution rate is $0.789 \mathrm{~g} / \mathrm{min}$.

After an activation period ( $3 \mathrm{~min}$ ), dissolution rate is $1.05 \mathrm{~g} / \mathrm{min}$. 
A small batch of recycled Mo material as $\mathrm{MoO}_{3}$ obtained using a precipitation method developed at Argonne [5], and ammonium heptamolybdate (AHM) derived by solvent extraction [6], was sent to ORNL. At ORNL this material was reduced to Mo metal using a two-stage process [7], producing $12 \times 1 \mathrm{~mm}$ sintered Mo disks for Argonne dissolution studies. Characteristics and dissolution rates of the sintered Mo disks are listed in Table 2.

Reduction of the AHM material led to an approximately $25-30 \%$ decrease in the sizes of obtained Mo particles. Finer AHM material with particle sizes of $131 \pm 30 \mu \mathrm{m}$ turned into $91 \pm 25 \mu \mathrm{m}$ Mo particles after reduction, and coarser AHM with particle sizes of $204 \pm 37 \mu \mathrm{m}$ turned into Mo particle sizes of $158 \pm 38 \mu \mathrm{m}$. Reduction of $\mathrm{MoO}_{3}$ material with particle sizes of $75-150 \mu \mathrm{m}$ led to Mo particle sizes of $125 \pm 24 \mu \mathrm{m}$ (ANL-0815-A) and $155 \pm 45 \mu \mathrm{m}$ (ANL-0815-B), which were agglomerates of much smaller particles, typically in the 1-10 $\mu \mathrm{m}$ range, and resulted in high-theoretical-density disks.

Disks made from recycled material did not start dissolving in room temperature $\mathrm{H}_{2} \mathrm{O}_{2}$ even after $15 \mathrm{~min}$. When $\mathrm{H}_{2} \mathrm{O}_{2}$ was preheated to $70^{\circ} \mathrm{C}$, the disks made from recycled AHM (ANL-1015) dissolved quickly, with dissolution rates of $0.33-0.75 \mathrm{~g} / \mathrm{min}$. There was no significant difference in dissolution rates for disks made from coarser or finer Mo material obtained after reduction of AHM.

The disks made after reduction of recycled $\mathrm{MoO}_{3}$ (ANL-815) dissolved much slower, and continuous heat (boiling $\mathrm{H}_{2} \mathrm{O}_{2}$ ) was required for complete dissolution. This is due to the very high density and low open porosity of sintered Mo disks that were produced from fine Mo material. 
TABLE 2 Characteristics and dissolution results for sintered Mo disks from recycled material in $40 \mathrm{~mL}$ of $50 \% \mathrm{H}_{2} \mathrm{O}_{2}$

\begin{tabular}{|c|c|c|c|c|c|c|c|c|c|c|c|}
\hline Material & No. & $\begin{array}{c}\text { Temp. } \\
\text { /time } \\
\left({ }^{\circ} \mathrm{C} / \mathrm{hr}\right)\end{array}$ & $\begin{array}{c}\text { Mass } \\
(\mathrm{g})\end{array}$ & $\begin{array}{l}\mathrm{TD}^{*} \\
(\%) \\
\end{array}$ & $\begin{array}{c}\text { Open } \\
\text { Porosity } \\
(\%)\end{array}$ & $\begin{array}{c}\text { Diameter } \\
(\mathrm{mm})\end{array}$ & $\begin{array}{c}\text { Thickness } \\
(\mathrm{mm})\end{array}$ & $\begin{array}{c}\text { Temp. } \\
\left({ }^{\circ} \mathrm{C}\right)\end{array}$ & $\begin{array}{l}\text { Diss. } \\
\text { Time } \\
(\mathrm{min}) \\
\end{array}$ & $\begin{array}{c}\text { Diss. } \\
\text { Rate } \\
(\mathrm{g} / \mathrm{min})\end{array}$ & $\begin{array}{c}\text { Particle Size } \\
\text { Recycled Mo/reduced } \mathrm{Mo}^{*}\end{array}$ \\
\hline ANL-1015A & $\mathrm{F} 1$ & $1500 / 1$ & 0.993 & 87.0 & 11.3 & 12.00 & 1.036 & 70 & 1.50 & 0.66 & $75-150 \mu \mathrm{m}$ AHM/91 $\pm 25 \mu \mathrm{m}$ Mo \\
\hline ANL-1015A & $\mathrm{F} 2$ & $1500 / 1$ & 1.003 & 86.9 & 11.6 & 12.00 & 1.034 & 70 & 1.67 & 0.60 & $75-150 \mu \mathrm{m}$ AHM/91 $\pm 25 \mu \mathrm{m} \mathrm{Mo}$ \\
\hline ANL-1015A & F3 & $1500 / 1$ & 0.996 & 87.4 & 10.8 & 11.99 & 1.033 & 70 & 1.33 & 0.75 & $75-150 \mu \mathrm{m}$ AHM/91 $\pm 25 \mu \mathrm{m}$ Mo \\
\hline ANL-1015A & F4 & $1600 / 4$ & 1.002 & 89.9 & 7.2 & 11.79 & 1.021 & 70 & 3.00 & 0.33 & $75-150 \mu \mathrm{m}$ AHM/91 $\pm 25 \mu \mathrm{m}$ Mo \\
\hline ANL-1015A & F5 & $1600 / 4$ & 1.009 & 90.7 & 7.2 & 11.76 & 1.041 & 70 & 2.67 & 0.38 & $75-150 \mu \mathrm{m}$ AHM/91 $\pm 25 \mu \mathrm{m} \mathrm{Mo}$ \\
\hline ANL-1015A & F6 & $1600 / 4$ & 1.000 & 91.4 & 7.0 & 11.76 & 1.011 & 70 & 2.33 & 0.43 & $75-150 \mu \mathrm{m}$ AHM/91 $\pm 25 \mu \mathrm{m} \mathrm{Mo}$ \\
\hline ANL-1015A & $\mathrm{C} 1$ & $1500 / 1$ & 1.001 & 86.9 & 10.6 & 12.05 & 1.029 & 70 & 1.33 & 0.75 & $150-400 \mu \mathrm{m} \mathrm{AHM} / 204 \pm 37 \mu \mathrm{m} \mathrm{Mo}$ \\
\hline ANL-1015A & $\mathrm{C} 2$ & $1500 / 1$ & 1.004 & 87.5 & 10.3 & 12.05 & 1.021 & 70 & 1.75 & 0.57 & $150-400 \mu \mathrm{m}$ AHM/204 $\pm 37 \mu \mathrm{m} \mathrm{Mo}$ \\
\hline ANL-1015A & $\mathrm{C} 3$ & $1500 / 1$ & 1.002 & 87.6 & 10.1 & 12.05 & 1.010 & 70 & 1.67 & 0.60 & $150-400 \mu \mathrm{m}$ AHM/204 $\pm 37 \mu \mathrm{m} \mathrm{Mo}$ \\
\hline ANL-1015A & $\mathrm{C} 4$ & $1600 / 4$ & 1.029 & 87.9 & 9.9 & 11.94 & 1.029 & 70 & N/A & $\mathrm{N} / \mathrm{A}$ & $150-400 \mu \mathrm{m}$ AHM/204 $\pm 37 \mu \mathrm{m} \mathrm{Mo}$ \\
\hline ANL-1015A & $\mathrm{C} 5$ & $1600 / 4$ & 1.012 & 89.1 & 8.9 & 11.94 & 1.006 & 70 & 2.25 & 0.45 & $150-400 \mu \mathrm{m}$ AHM/204 $\pm 37 \mu \mathrm{m} \mathrm{Mo}$ \\
\hline ANL-1015A & C6 & $1600 / 4$ & 0.995 & 88.3 & 9.8 & 11.94 & 1.006 & 70 & 2.17 & 0.46 & $150-400 \mu \mathrm{m}$ AHM/204 $\pm 37 \mu \mathrm{m} \mathrm{Mo}$ \\
\hline ANL-815-A & A1 & $1600 / 4$ & 1.001 & 93.3 & 0.0 & 11.46 & 1.049 & 100 & 6.00 & 0.17 & $75-150 \mu \mathrm{m} \mathrm{MoO}_{3} / 125-155 \mu \mathrm{m} \mathrm{Mo}^{+}$ \\
\hline ANL-815-A & A2 & $1600 / 4$ & 1.004 & 93.7 & 0.1 & 11.56 & 1.016 & 100 & 9.00 & 0.11 & $75-150 \mu \mathrm{m} \mathrm{MoO}_{3} / 125-155 \mu \mathrm{m} \mathrm{Mo}^{+}$ \\
\hline ANL-815-A & A3 & $1600 / 4$ & 1.007 & 93.7 & 0.6 & 11.60 & 1.016 & 100 & 7.75 & 0.13 & $75-150 \mu \mathrm{m} \mathrm{MoO}_{3} / 125-155 \mu \mathrm{m} \mathrm{Mo}^{+}$ \\
\hline ANL-815-B & A1 & $1600 / 4$ & 1.015 & 96.1 & 0.7 & 11.79 & 0.978 & 100 & 17.0 & 0.060 & $75-150 \mu \mathrm{m} \mathrm{MoO}_{3} / 125-155 \mu \mathrm{m} \mathrm{Mo}^{+}$ \\
\hline ANL-815-B & A2 & $1600 / 4$ & 1.016 & 96.8 & 0.4 & 11.76 & 0.965 & 100 & 15.3 & 0.067 & $75-150 \mu \mathrm{m} \mathrm{MoO}_{3} / 125-155 \mu \mathrm{m} \mathrm{Mo}^{+}$ \\
\hline ANL-815-B & A3 & $1600 / 4$ & 0.997 & 96.6 & 0.6 & 11.79 & 0.932 & 100 & 13.5 & 0.074 & $75-150 \mu \mathrm{m} \mathrm{MoO}_{3} / 125-155 \mu \mathrm{m} \mathrm{Mo}^{+}$ \\
\hline
\end{tabular}

* $\mathrm{TD}=$ theoretical density, $\mathrm{AHM}=$ ammonium heptamolybdate

Most particles were agglomerates of 1-10 $\mu \mathrm{m}$ Mo particles. 


\section{Laser-Melt 3D-Printed Mo Disks}

Characteristics of laser-melt 3D-printed Mo disks and dissolution times are listed in Table 3. Dissolution rates were in the range of $\sim 0.1-0.25 \mathrm{~g} / \mathrm{min}$. In general, laser-melt 3Dprinted disks dissolved more slowly than sintered Mo disks. After 1-2 min of dissolution, the disks broke down into very small pieces. Complete dissolution of the smaller particles took several additional minutes and heat provided by a hot plate. Some of the fine particles looked like tiny wires (disk Mo-45-16-9; see Figure 3).

TABLE 3 Characteristics and dissolution results for laser-melt 3D-printed Mo disks in 40 mL of $50 \% \mathrm{H}_{2} \mathrm{O}_{2}$

\begin{tabular}{|c|c|c|c|c|c|c|c|c|}
\hline Disk I.D. & $\begin{array}{c}\text { Mass } \\
(\mathrm{g})\end{array}$ & $\begin{array}{c}\text { Theor. } \\
\text { Density } \\
(\%)\end{array}$ & $\begin{array}{c}\text { Open } \\
\text { Porosity } \\
(\%)\end{array}$ & $\begin{array}{l}\text { Diameter } \\
(\mathrm{mm})\end{array}$ & $\begin{array}{l}\text { Thickness } \\
(\mathrm{mm})\end{array}$ & $\begin{array}{c}\text { Temperature } \\
\left({ }^{\circ} \mathrm{C}\right)\end{array}$ & $\begin{array}{l}\text { Diss. } \\
\text { Time } \\
\text { (min) }\end{array}$ & $\begin{array}{l}\text { Diss. } \\
\text { Rate } \\
(\mathrm{g} / \mathrm{min})\end{array}$ \\
\hline Mo45-16-3 & 1.6470 & 90.0 & 9.8 & 12.40 & 1.90 & 70 & 10.00 & 0.165 \\
\hline Мo45-16-9 & 1.7380 & 89.0 & 10.4 & 12.40 & 1.90 & 70 & 18.00 & 0.097 \\
\hline Mo45-23-1 & 1.2660 & 89.5 & 8.5 & 12.40 & 1.50 & 24 & 6.00 & $0.211^{*}$ \\
\hline Mo45-23-2 & 1.2840 & 91.0 & 7.5 & 12.40 & 1.50 & 70 & + & + \\
\hline Mo45-23-7 & 1.272 & 90.3 & 6.7 & 12.40 & 1.50 & 70 & 5.00 & 0.254 \\
\hline
\end{tabular}

* Dissolution started in $24^{\circ} \mathrm{C}_{2} \mathrm{O}_{2}$; however, fine particles had to be dissolved with additional heat using hot plate.

+ It was difficult to determine the exact time of dissolution because of profuse bubbles.

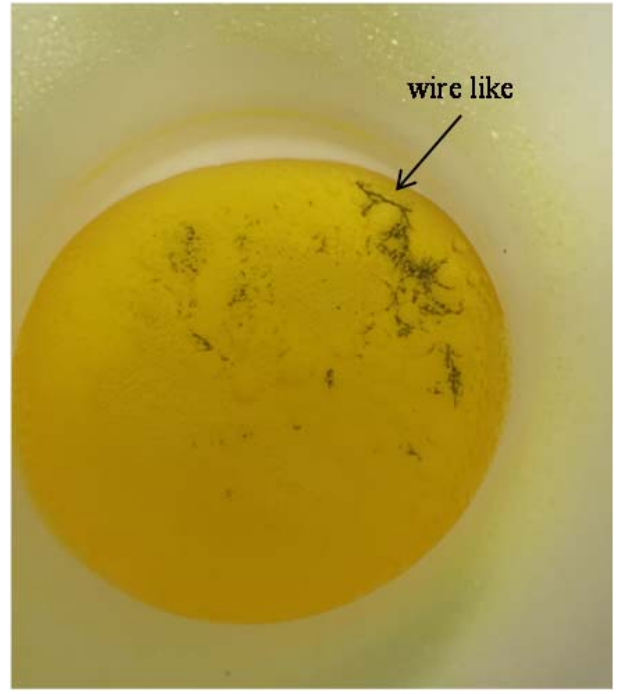

FIGURE 3 Photograph of residual small particles that form during the dissolution of laser-melt 3D-printed Mo disks
Based on a very limited number of disks, the dependency of laser-melt disk dissolution rates on open porosity was observed to be reversed compared with sintered disks. Disks with the highest degree of open porosity $(10.4 \%$ ) dissolved the slowest (below $0.1 \mathrm{~g} / \mathrm{min}$ ), while disks with the lowest open porosity $(6.7 \%)$ dissolved the fastest $(0.254 \mathrm{~g} / \mathrm{min})$. A possible explanation could be that the laser-melt disks with large open porosity contain fused particles with different macro- and microstructures that require a longer time to dissolve.

\section{9 mm Sintered Mo Disks}

All disks provided by ORNL were sintered at $1,600^{\circ} \mathrm{C}$ for $4 \mathrm{hr}$ and pressed at $138 \mathrm{ksi}$. Characteristics of the sintered Mo disks and dissolution times are listed in Table 4. To determine the best conditions for dissolution, an S100-29 disk was dissolved first by adding $110 \mathrm{~mL}$ of $\mathrm{H}_{2} \mathrm{O}_{2}$ preheated to $70^{\circ} \mathrm{C}$. After 9.5 min, the solution turned 
orange in color, indicating that most of the $\mathrm{H}_{2} \mathrm{O}_{2}$ was consumed, and the dissolution slowed down significantly. Then, $50 \mathrm{~mL}$ of additional $\mathrm{H}_{2} \mathrm{O}_{2}$ preheated to $70^{\circ} \mathrm{C}$ was added to finish the dissolution. On the basis of this initial test, further dissolutions were performed by adding $100 \mathrm{~mL}$ of $\mathrm{H}_{2} \mathrm{O}_{2}$ preheated to $70^{\circ} \mathrm{C}$, draining the solution after $5.5-6 \mathrm{~min}$, and adding $50 \mathrm{~mL}$ to finish the dissolution.

TABLE 4 Characteristics and dissolution results for sintered Mo disks in $150 \mathrm{~mL}$ of $50 \% \mathrm{H}_{2} \mathrm{O}_{2}$

\begin{tabular}{lcccccccc}
\hline & & & & & & & & Diss. \\
Disk* & Mass (g) & $\begin{array}{c}\text { Theor. } \\
\text { Density } \\
(\%)\end{array}$ & $\begin{array}{c}\text { Open } \\
\text { Porosity } \\
(\%)\end{array}$ & $\begin{array}{c}\text { Diameter } \\
(\mathrm{mm})\end{array}$ & $\begin{array}{c}\text { Thickness } \\
(\mathrm{mm})\end{array}$ & $\begin{array}{c}\text { Temp. } \\
\left({ }^{\circ} \mathrm{C}\right)\end{array}$ & $\begin{array}{c}\text { Time } \\
(\mathrm{min})\end{array}$ & $\begin{array}{c}\text { Rate } \\
(\mathrm{g} / \mathrm{min})\end{array}$ \\
\hline S25-36 & 12.1436 & 90.5 & 2.2 & 29.40 & 1.95 & 70 & 8.750 & 1.388 \\
S25-37 & 12.1087 & 90.5 & 2.0 & 29.40 & 1.92 & 70 & 9.000 & 1.345 \\
S25-38 & 12.0875 & 90.7 & 1.0 & 29.40 & 1.92 & 70 & + & + \\
S25-39 & 12.1169 & 90.7 & 1.7 & 29.40 & 1.94 & 70 & 8.500 & 1.426 \\
S25-40 & 12.0544 & 90.7 & 1.6 & 29.40 & 1.90 & 70 & 8.750 & 1.378 \\
& & & & & & & & \\
S50-41 & 12.1019 & 90.9 & 2.8 & 29.40 & 1.95 & 70 & 5.00 & 0.254 \\
S50-42 & 12.1192 & 90.6 & 2.7 & 29.40 & 1.95 & 70 & 7.666 & 1.579 \\
S50-43 & 12.0707 & 90.7 & 2.7 & 29.40 & 1.92 & 70 & 9.000 & 1.347 \\
S50-44 & 12.0866 & 90.5 & 2.8 & 29.40 & 1.93 & 70 & 9.000 & 1.341 \\
S50-45 & 12.0820 & 90.6 & 3.0 & 29.40 & 1.94 & 70 & 8.00 & 1.511 \\
& & & & & & & & \\
S100-29 & 12.0330 & 89.0 & 6.1 & 29.50 & 1.94 & 70 & 8.50 & 1.421 \\
S100-30 & 12.0210 & 89.0 & 6.3 & 29.50 & 1.94 & 70 & 11.00 & 1.094 \\
S100-31 & 12.0722 & 89.7 & 5.3 & 29.50 & 1.99 & 70 & 7.50 & 1.603 \\
S100-32 & 12.1604 & 89.1 & 5.7 & 29.50 & 1.97 & 70 & 8.00 & 1.509 \\
S100-33 & 12.0416 & 89.1 & 5.9 & 29.50 & 1.96 & 24 & $10.50^{++}$ & $1.158^{++}$ \\
S100-34 & 12.0900 & 89.4 & 5.5 & 29.50 & 1.96 & 70 & 15.50 & 0.777 \\
S100-35 & 12.0448 & 89.5 & 5.2 & 29.50 & 1.95 & 24 & $8.00^{\S}$ & $1.51^{\S}$ \\
\hline
\end{tabular}

* Different amounts of ethylene bis stearamide (EBS) lubricant were used: from $0.25 \%$ to $1 \%$. For disks marked S25, $0.25 \%$ EBS was used to make the disks; for 0.5\% EBS, the disks were marked S50; and for 1\% EBS, the disks were marked S100.

+ Most of the disk dissolved in $\sim 13 \mathrm{~min}$; the remaining portion would not dissolve even after an additional $20 \mathrm{~min}$.

++ Started to dissolve after adding $100 \mathrm{~mL} \mathrm{H}_{2} \mathrm{O}_{2}$ - activation period $8 \mathrm{~min}$, dissolution rate $1.7 \mathrm{~g} / \mathrm{min}$.

$\S$ Started to dissolve after adding $30 \mathrm{~mL} \mathrm{H}_{2} \mathrm{O}_{2}$ - activation period $4.5 \mathrm{~min}$, dissolution rate $1.46 \mathrm{~g} / \mathrm{min}$.

Typical dissolution rates were in the range of 1.34-1.6 g/min. Disk S25-38, with 1\% open porosity, didn't completely dissolve in $150 \mathrm{~mL}$ of $\mathrm{H}_{2} \mathrm{O}_{2}$ even after prolonged time. Although the majority of the disks ( $>95 \%$ of mass) dissolved in $\sim 13 \mathrm{~min}$, the remaining portion of the disks did not completely dissolve, even after an additional $20 \mathrm{~min}$ (without additional heat 
provided). Disks that were dissolved with room-temperature $\mathrm{H}_{2} \mathrm{O}_{2}$ required an activation period before they started to dissolve quickly, usually after the $\mathrm{H}_{2} \mathrm{O}_{2}$ reached $60^{\circ} \mathrm{C}$ (without heating). Less $\mathrm{H}_{2} \mathrm{O}_{2}$ at the beginning ( $30 \mathrm{~mL}$ for disk $\left.\mathrm{S} 100-35\right)$ led to shorter activation periods (4.5 min), compared with an activation period of 8 min when $100 \mathrm{~mL}$ of $\mathrm{H}_{2} \mathrm{O}_{2}$ was used. With less mass to absorb the heat from the reaction, the solution warmed up much quicker. It appears that using $29 \times 2 \mathrm{~mm}$ disks for a target should not be a problem from the dissolution point of view.

Table 5 summarizes the results from dissolution of disks with $\sim 29 \mathrm{~mm}$ diameter and various thicknesses: $0.5,1,1.5$, and $2 \mathrm{~mm}$. All disks were manufactured from commercial Mo materials (Climax Molybdenum or H.C. Starck). All disks were dissolved in $50 \% \mathrm{H}_{2} \mathrm{O}_{2}$ preheated to $70^{\circ} \mathrm{C}$. Generally, $\sim 13 \mathrm{~mL}$ of $\mathrm{H}_{2} \mathrm{O}_{2}$ was used per gram of Mo material. Based on the dissolution of a few thicker disks, it seemed the dissolution rate was slightly higher for thicker disks than thinner disks; however, this was due to the fact that thinner and lighter disks floated on top of froth during the dissolution (Figure 4a), which significantly limited their contact with solution. Thicker and heavier disks remained at the bottom of the reaction vessel for most of the dissolution despite the very vigorous reaction (Figure $4 \mathrm{~b}$ ), forming significant volume of froth ( $\sim 70 \%$ of $2 \mathrm{~L}$ vessel), and only started to float toward the end of dissolution when the mass of the disks was no longer heavy enough to keep the material from floating.

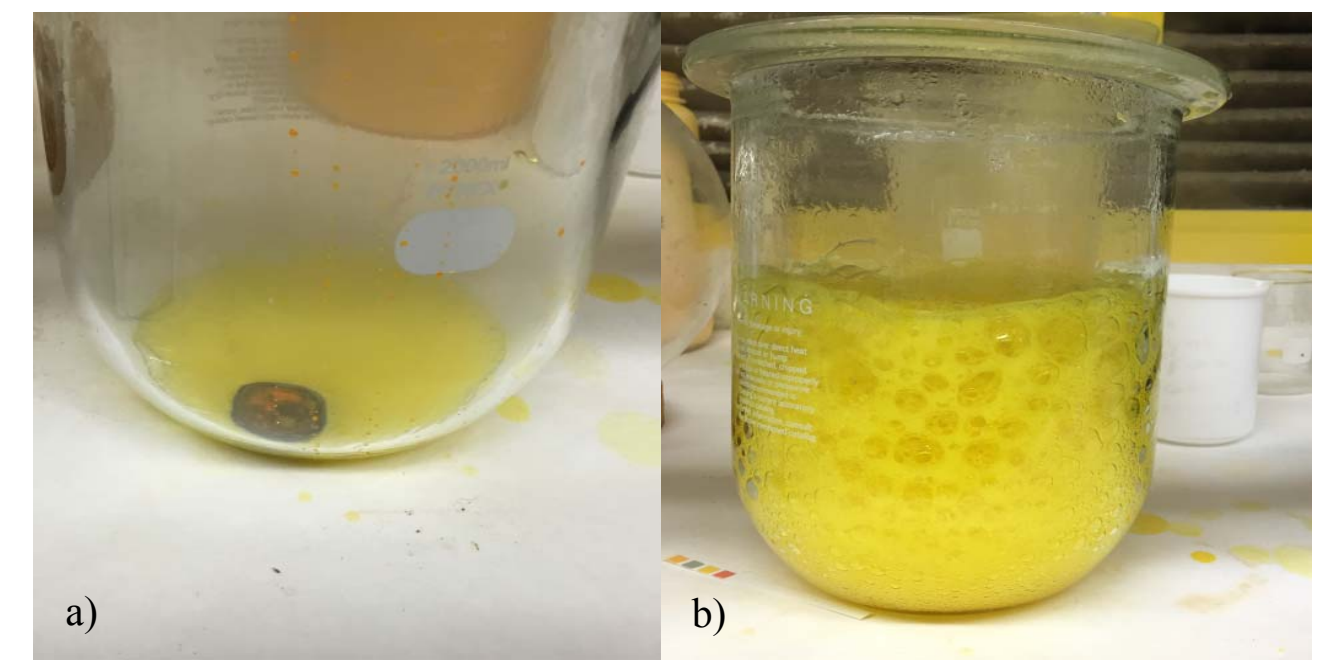

FIGURE 4 (a) Floating $29 \times 0.5 \mathrm{~mm}$ sintered Mo disks during dissolution in $40 \mathrm{~mL}$ of $50 \% \mathrm{H}_{2} \mathrm{O}_{2}$; (b) Formation of froth in $2 \mathrm{~L}$ reaction vessel during the dissolution of $12 \mathrm{~g}$ of $29 \times 2 \mathrm{~mm}$ sintered Mo disks in $150 \mathrm{~mL}$ of $50 \% \mathrm{H}_{2} \mathrm{O}_{2}$ 
TABLE 5 Characteristics and dissolution results for sintered Mo disks in $50 \% \mathrm{H}_{2} \mathrm{O}_{2}$ preheated to $70^{\circ} \mathrm{C}$

\begin{tabular}{|c|c|c|c|c|c|c|c|c|c|c|c|c|c|}
\hline Disk I.D. & Material & $\begin{array}{c}\text { Press } \\
(\mathrm{ksi})\end{array}$ & $\begin{array}{c}\text { Sinter } \\
\left({ }^{\circ} \mathrm{C} / \mathrm{hr}\right)\end{array}$ & $\begin{array}{c}\text { Mass } \\
(\mathrm{g})\end{array}$ & $\begin{array}{l}\text { Density } \\
\left(\mathrm{g} / \mathrm{cm}^{3}\right)\end{array}$ & $\begin{array}{c}\text { TD } \\
\text { Sintered } \\
(\%)\end{array}$ & $\begin{array}{c}\text { Open } \\
\text { Porosity } \\
(\%)\end{array}$ & $\begin{array}{c}\text { Diameter } \\
(\mathrm{mm})\end{array}$ & $\begin{array}{c}\text { Thickness } \\
(\mathrm{mm})\end{array}$ & $\begin{array}{l}\text { Diss. } \\
\text { Time } \\
(\mathrm{min})\end{array}$ & $\begin{array}{c}\text { Diss. } \\
\text { Rate } \\
(\mathrm{g} / \mathrm{min})\end{array}$ & $\begin{array}{l}50 \% \\
\mathrm{H}_{2} \mathrm{O}_{2} \\
(\mathrm{~mL})\end{array}$ & $\begin{array}{c}\mathrm{H}_{2} \mathrm{O}_{2} / \\
\mathrm{Mo} \\
(\mathrm{mL} / \mathrm{g})\end{array}$ \\
\hline PMS-1 & Climax PM $+0.5 \% \mathrm{SA}$ & 100 & $1600 / 4$ & 3.0797 & 9.14 & 89.6 & 7.3 & 28.8 & 0.53 & 1.5 & 2.05 & 40 & 13.0 \\
\hline PMS-2 & Climax PM $+0.5 \% \mathrm{SA}$ & 100 & $1600 / 4$ & 3.0923 & 9.08 & 89 & 9.1 & 28.8 & 0.54 & 1.75 & 1.77 & 40 & 12.9 \\
\hline PMS-3 & Climax PM $+0.5 \% \mathrm{SA}$ & 100 & $1600 / 4$ & 3.0725 & 8.95 & 87.7 & 8.5 & 28.8 & 0.53 & 1.633 & 1.88 & 40 & 13.0 \\
\hline PMA5-3 & Climax PM $+0.5 \%$ EBS & 100 & $1600 / 2$ & 3.0996 & 9.04 & 88.6 & 9.3 & 29 & 0.53 & 1.5 & 2.07 & 40 & 12.9 \\
\hline PMA5-24 & Climax PM $+0.5 \%$ EBS & 100 & $1600 / 4$ & 6.0576 & 8.99 & 88.1 & 6.7 & 29.3 & 1 & 2.75 & 2.20 & 60 & 9.9 \\
\hline PMA5-26 & Climax PM $+0.5 \%$ EBS & 100 & $1600 / 4$ & 6.0533 & 9.00 & 88.2 & 6.9 & 29.3 & 1 & 2.25 & 2.69 & 80 & 13.2 \\
\hline PMA5-27 & Climax PM $+0.5 \%$ EBS & 100 & $1600 / 4$ & 9.0418 & 8.92 & 87.5 & 7.7 & 29.3 & 1.49 & 4 & 2.26 & 100 & 11.1 \\
\hline PMA5-28 & Climax PM $+0.5 \%$ EBS & 100 & $1600 / 4$ & 9.0453 & 8.92 & 87.5 & 7.8 & 29.3 & 1.5 & 3.33 & 2.72 & 100 & 11.1 \\
\hline PM5A-31 & Climax PM $+0.5 \%$ EBS & 100 & $1600 / 4$ & 12.0738 & 8.90 & 87.3 & 7.9 & 29.4 & 2 & 5.5 & 2.20 & 150 & 12.4 \\
\hline PMA5-32 & Climax PM $+0.5 \%$ EBS & 100 & $1600 / 4$ & 12.0781 & 8.91 & 87.4 & 7.9 & 29.4 & 1.99 & 4.17 & 2.90 & 150 & 12.4 \\
\hline PMA-13 & Climax PM $+0.25 \%$ EBS & 100 & $1600 / 4$ & 3.0899 & 9.23 & 90.5 & 2.4 & 28.8 & 0.52 & 2 & 1.54 & 40 & 12.9 \\
\hline PMA-27 & Climax PM $+0.25 \%$ EBS & 100 & $1400 / 2$ & 3.1001 & 8.71 & 85.4 & --- & 29.2 & 0.53 & 1.33 & 2.33 & 40 & 12.9 \\
\hline PMA-40 & Climax PM $+0.25 \%$ EBS & 100 & $1600 / 4$ & 3.0946 & 9.1 & 89.2 & 6.2 & 28.8 & 0.53 & 1.75 & 1.77 & 40 & 12.9 \\
\hline PMA-41 & Climax PM $+0.25 \%$ EBS & 100 & $1600 / 4$ & 3.0952 & 9.06 & 88.8 & 6.3 & 28.8 & 0.54 & 1.75 & 1.77 & 40 & 12.9 \\
\hline PMA-43 & Climax PM $+0.25 \%$ EBS & 100 & $1600 / 4$ & 3.0975 & 9.09 & 89.1 & 6.4 & 28.8 & 0.53 & 1.72 & 1.80 & 40 & 12.9 \\
\hline PMA-44 & Climax PM $+0.25 \%$ EBS & 100 & $1600 / 4$ & 3.0893 & 9.05 & 88.7 & 6.4 & 28.8 & 0.53 & 1.78 & 1.74 & 40 & 12.9 \\
\hline PMA-46 & Climax PM $+0.25 \%$ EBS & 100 & $1600 / 4$ & 3.0732 & 9.07 & 88.9 & 6.6 & 28.8 & 0.53 & 1.75 & 1.76 & 40 & 13.0 \\
\hline PM-2 & Climax PM & 100 & $1500 / 16$ & 3.0804 & 7.86 & 77.1 & 20.9 & 27.3 & 0.73 & 1.33 & 2.32 & 40 & 13.0 \\
\hline PM-3 & Climax PM & 100 & $1500 / 1$ & 3.0818 & 7.10 & 69.6 & 28.9 & 28.3 & 0.7 & 1.17 & 2.63 & 40 & 13.0 \\
\hline PM-4 & Climax PM & 100 & $1500 / 1$ & 3.1083 & 8.68 & 85.1 & 12.7 & 29.3 & 0.55 & 1.33 & 2.34 & 40 & 12.9 \\
\hline PM-5 & Climax PM & 100 & $1500 / 1$ & 3.0806 & 8.54 & 83.7 & 14.3 & 29.3 & 0.55 & 1.33 & 2.32 & 40 & 13.0 \\
\hline PM-7 & Climax PM & 100 & $1600 / 4$ & 3.1087 & 9.08 & 89 & 6.6 & 28.8 & 0.54 & 1.75 & 1.78 & 40 & 12.9 \\
\hline PM-9 & Climax PM & 100 & $1600 / 1$ & 3.0834 & 8.87 & 87 & 9.9 & 29.1 & 0.54 & 1.33 & 2.32 & 40 & 13.0 \\
\hline PM-10 & Climax PM & 100 & $1600 / 1$ & 3.1099 & 8.88 & 87.1 & 9.6 & 29.1 & 0.55 & 1.417 & 2.19 & 40 & 12.9 \\
\hline PM-11 & Climax PM & 100 & $1500 / 1$ & 3.0844 & 8.76 & 85.9 & 12.2 & 29.3 & 0.54 & 1.417 & 2.18 & 40 & 13.0 \\
\hline PM-12 & Climax PM & 100 & $1600 / 4$ & 3.0989 & 9.03 & 88.5 & 8.6 & 28.7 & 0.54 & 1.58 & 1.96 & 40 & 12.9 \\
\hline PM-14 & Climax PM & 145 & $1600 / 2$ & 3.0966 & 9.36 & 91.8 & 3.4 & 29.3 & 0.5 & 1.75 & 1.77 & 40 & 12.9 \\
\hline PM-15 & Climax PM & 145 & $1600 / 4$ & 3.1242 & 9.47 & 92.8 & 1 & 29.1 & 0.52 & 2 & 1.56 & 40 & 12.8 \\
\hline PM-16 & Climax PM & 145 & $1600 / 2$ & 3.0198 & 9.40 & 92.2 & 3.9 & 29.3 & 0.55 & 2 & 1.51 & 40 & 13.2 \\
\hline
\end{tabular}


TABLE 5 (Cont.)

\begin{tabular}{|c|c|c|c|c|c|c|c|c|c|c|c|c|c|}
\hline Disk I.D. & Material & $\begin{array}{c}\text { Press } \\
(\mathrm{ksi})\end{array}$ & $\begin{array}{l}\text { Sinter } \\
\left({ }^{\circ} \mathrm{C} / \mathrm{hr}\right) \\
\end{array}$ & $\begin{array}{c}\text { Mass } \\
(\mathrm{g}) \\
\end{array}$ & $\begin{array}{l}\text { Density } \\
\left(\mathrm{g} / \mathrm{cm}^{3}\right)\end{array}$ & $\begin{array}{c}\text { TD } \\
\text { Sintered } \\
(\%)\end{array}$ & $\begin{array}{c}\text { Open } \\
\text { Porosity } \\
(\%)\end{array}$ & $\begin{array}{c}\text { Diameter } \\
(\mathrm{mm})\end{array}$ & $\begin{array}{c}\text { Thickness } \\
(\mathrm{mm})\end{array}$ & $\begin{array}{l}\text { Diss. } \\
\text { Time } \\
(\mathrm{min}) \\
\end{array}$ & $\begin{array}{c}\text { Diss. } \\
\text { Rate } \\
\text { (g/min) }\end{array}$ & $\begin{array}{l}50 \% \\
\mathrm{H}_{2} \mathrm{O}_{2} \\
(\mathrm{~mL}) \\
\end{array}$ & $\begin{array}{c}\mathrm{H}_{2} \mathrm{O}_{2} / \\
\mathrm{Mo} \\
(\mathrm{mL} / \mathrm{g}) \\
\end{array}$ \\
\hline S5-1 & Starck SD $+0.5 \% \mathrm{EBS}$ & 100 & $1600 / 4$ & 3.1545 & 8.82 & 86.5 & 9 & 28.9 & 0.55 & 1.5 & 2.10 & 40 & 12.7 \\
\hline S5-2 & Starck SD $+0.5 \% \mathrm{EBS}$ & 100 & $1600 / 4$ & 3.1481 & 8.8 & 86.3 & 9.3 & 28.9 & 0.56 & 1.5 & 2.10 & 40 & 12.7 \\
\hline S5-4 & Starck SD $+0.5 \% \mathrm{EBS}$ & 100 & $1600 / 4$ & 3.12 & 8.81 & 86.4 & 9.4 & 28.9 & 0.55 & 1.42 & 2.20 & 40 & 12.8 \\
\hline S5-5 & Starck SD $+0.5 \% \mathrm{EBS}$ & 100 & $1600 / 4$ & 3.1447 & 8.89 & 87.2 & 8.9 & 28.9 & 0.55 & 1.5 & 2.10 & 40 & 12.7 \\
\hline S5-6 & Starck SD $+0.5 \%$ EBS & 131 & $1600 / 4$ & 3.0303 & 9.08 & 89 & 6.5 & 29.3 & 0.5 & 1.33 & 2.28 & 40 & 13.2 \\
\hline S5-8 & Starck SD $+0.5 \% \mathrm{EBS}$ & 131 & $1600 / 4$ & 3.0393 & 9.03 & 88.5 & 6.8 & 29.3 & 0.51 & 1.42 & 2.14 & 40 & 13.2 \\
\hline S5-9 & Starck SD $+0.5 \% \mathrm{EBS}$ & 148 & $1600 / 4$ & 3.0263 & 9.20 & 90.2 & 4.7 & 29.4 & 0.5 & 1.5 & 2.02 & 40 & 13.2 \\
\hline S5-10 & Starck SD $+0.5 \%$ EBS & 148 & $1600 / 4$ & 3.0511 & 9.22 & 90.4 & 4.4 & 29.4 & 0.5 & 1.58 & 1.93 & 40 & 13.1 \\
\hline S5-11 & Starck SD $+0.5 \%$ EBS & 148 & $1600 / 4$ & 3.0234 & 9.21 & 90.3 & 4.4 & 29.5 & 0.49 & 1.75 & 1.73 & 40 & 13.2 \\
\hline S5-12 & Starck SD $+0.5 \% \mathrm{EBS}$ & 123 & $1600 / 4$ & 3.0262 & 8.97 & 87.9 & 7.6 & 29.3 & 0.51 & 1.5 & 2.02 & 40 & 13.2 \\
\hline S5-13 & Starck SD $+0.5 \% \mathrm{EBS}$ & 123 & $1600 / 4$ & 3.0208 & 8.95 & 87.7 & 7.6 & 29.3 & 0.51 & 1.5 & 2.01 & 40 & 13.2 \\
\hline S5-15 & Starck SD $+0.5 \% \mathrm{EBS}$ & 123 & $1600 / 4$ & 3.0228 & 8.93 & 87.5 & 8 & 29.3 & 0.51 & 1.42 & 2.13 & 40 & 13.2 \\
\hline S5-16 & Starck SD $+0.5 \% \mathrm{EBS}$ & 123 & $1600 / 4$ & 3.0302 & 8.89 & 87.2 & 8.4 & 29.3 & 0.51 & 1.5 & 2.02 & 40 & 13.2 \\
\hline S5-17 & Starck SD $+0.5 \%$ EBS & 131 & $1600 / 4$ & 3.0069 & 9.07 & 89 & - & 29.4 & 0.49 & 1.5 & 2.00 & 40 & 13.3 \\
\hline S5-18 & Starck SD $+0.5 \%$ EBS & 131 & $1600 / 4$ & 3.028 & 9.15 & 89.7 & - & 29.4 & 0.49 & 1.33 & 2.28 & 40 & 13.2 \\
\hline S5-22 & Starck SD $+0.5 \%$ EBS & 137 & $1600 / 4$ & 3.0078 & 8.80 & 86.3 & - & 29.5 & 0.5 & 1.42 & 2.12 & 40 & 13.3 \\
\hline S5-23 & Starck SD $+0.5 \%$ EBS & 137 & $1600 / 4$ & 3.0053 & 8.84 & 86.7 & - & 29.5 & 0.5 & 1.42 & 2.12 & 40 & 13.3 \\
\hline S5-24 & Starck SD $+0.5 \% \mathrm{EBS}$ & 137 & $1600 / 4$ & 2.984 & 8.96 & 87.8 & - & 29.5 & 0.49 & 1.58 & 1.89 & 40 & 13.4 \\
\hline S5-26 & Starck SD $+0.5 \% \mathrm{EBS}$ & 137 & $1600 / 4$ & 3.0036 & 8.97 & 87.9 & - & 29.5 & 0.49 & 1.42 & 2.12 & 40 & 13.3 \\
\hline S5-27 & Starck SD $+0.5 \%$ EBS & 145 & $1600 / 4$ & 3.0225 & 8.97 & 87.9 & - & 29.5 & 0.49 & 1.58 & 1.91 & 40 & 13.2 \\
\hline S5-28 & Starck SD $+0.5 \%$ EBS & 145 & $1600 / 4$ & 3.0027 & 9.00 & 88.2 & - & 29.5 & 0.49 & 1.5 & 2.00 & 40 & 13.3 \\
\hline S5-30 & Starck SD $+0.5 \%$ EBS & 145 & $1600 / 4$ & 3.0096 & 9.09 & 89.1 & - & 29.5 & 0.48 & 1.5 & 2.01 & 40 & 13.3 \\
\hline S5-31 & Starck SD + 0.5\%EBS & 145 & $1600 / 4$ & 3.0106 & 9.28 & 91 & - & 29.5 & 0.47 & 1.42 & 2.12 & 40 & 13.3 \\
\hline RTP5-9 & Starck SD $+0.25 \%$ EBS & 87.5 & $1600 / 4$ & 3.0084 & 8.97 & 87.9 & - & 28.7 & 0.52 & 1.83 & 1.64 & 40 & 13.3 \\
\hline RTP5-10 & Starck SD $+0.25 \%$ EBS & 87.5 & $1600 / 4$ & 2.9906 & 9.23 & 90.5 & - & 28.7 & 0.5 & 1.75 & 1.71 & 40 & 13.4 \\
\hline S2-3 & Starck SD $+0.25 \%$ EBS & 100 & $1600 / 4$ & 3.1297 & 8.60 & 84.3 & 7.8 & 28.9 & 0.55 & 1.5 & 2.09 & 40 & 12.8 \\
\hline S2-4 & Starck SD $+0.25 \% \mathrm{EBS}$ & 100 & $1600 / 4$ & 3.0964 & 8.73 & 85.6 & 8.4 & 28.9 & 0.54 & 1.42 & 2.18 & 40 & 12.9 \\
\hline
\end{tabular}




\section{OPTIMIZATION OF LARGE-SCALE DISSOLUTION OF SINTERED MO DISKS}

\section{Experimental}

Sintered Mo disks received from NorthStar $(26 \times 1 \mathrm{~mm})$ and ORNL $(29 \times 0.5 \mathrm{~mm})$ were dissolved using $50 \% \mathrm{H}_{2} \mathrm{O}_{2}$ in $600 \mathrm{~g}$ batches in a glass reaction vessel. $\mathrm{H}_{2} \mathrm{O}_{2}(50 \%)$ stabilized with Sn (Aqua Solutions, Inc., Technical grade, H30504L) was used for these dissolutions. Disks were placed into a $4 \mathrm{~L}$ dissolution vessel with a glass frit on the bottom (Figure 5).

The dissolution apparatus is shown in Figure 6. All parts of dissolution apparatus are identified and referenced in the Appendix A. The apparatus consists of a $4 \mathrm{~L}$ dissolution vessel with glass-fritted filter and valved-drain (stopcock) at the bottom to allow for draining dissolved portions of Mo material into an evaporation vessel by vacuum. A Julabo FL 1701 with cooling capacities of $1.7 \mathrm{~kW}$ at $20^{\circ} \mathrm{C}, 1.1 \mathrm{~kW}$ at $0^{\circ} \mathrm{C}, 0.85 \mathrm{~kW}$ at $-10^{\circ} \mathrm{C}$, and $0.4 \mathrm{~kW}$ at $-20^{\circ} \mathrm{C}$ and flow rate capacity of $23 \mathrm{~L} / \mathrm{min}$ was used. However, it should be noted that due to small tubing connections (11 mm at the largest serration) and small internal diameter of the condenser coils, the actual coolant (50\% ethylene glycol) flowrate through the system was $0.32 \mathrm{~L} / \mathrm{min}$.

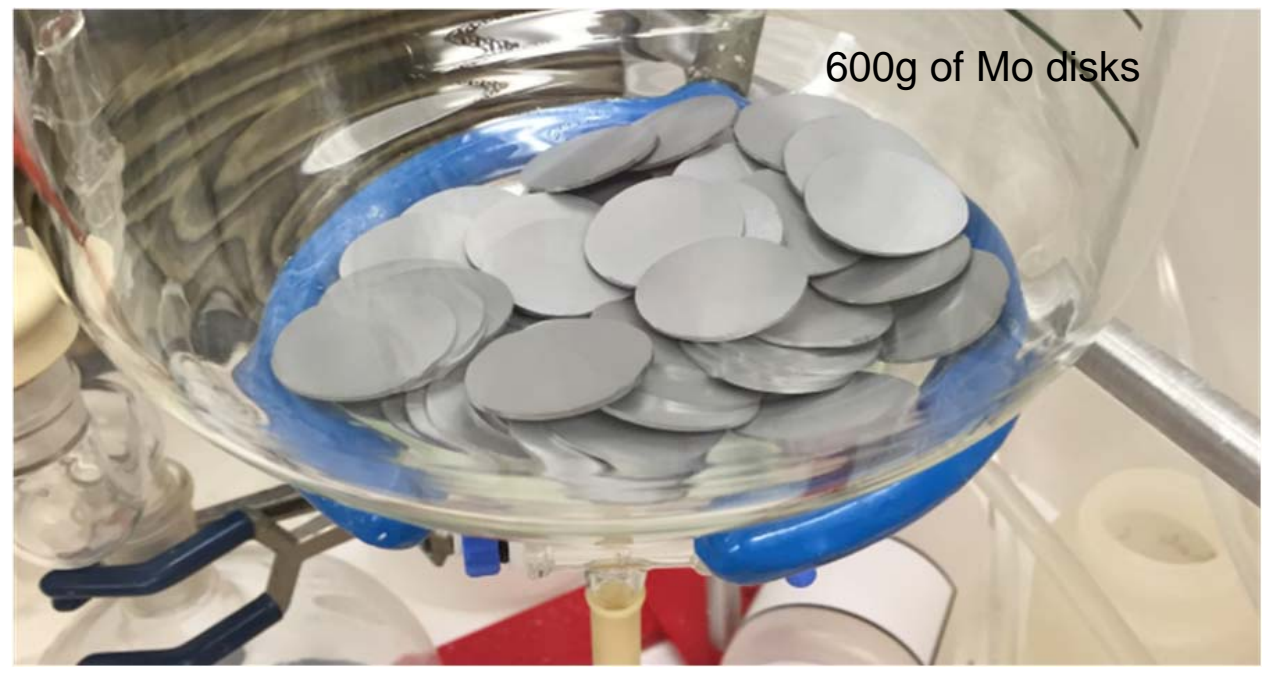

FIGURE 5 Photo of $600 \mathrm{~g}$ of sintered Mo disks placed inside $4 \mathrm{~L}$ dissolution vessel

Hydrogen peroxide was slowly added from the top of the vessel using a peristaltic pump. Shortly after addition of $\mathrm{H}_{2} \mathrm{O}_{2}$, vigorous dissolution of the Mo disks began, producing yellowcolored froth and solution and significant bubble formation due to heat produced from the reaction. Water vapor produced from the reaction was condensed using three high-efficiency condensers (Chemglass CG-1215-C) and collected in collection flask. The condensers were used to replace the lower-efficiency condensers (used in the previous system [1]) in order to trap the majority of vapors evolved during the dissolution process. Here the evolved gases flowed up and out of the dissolution vessel to the high-efficiency condensers in two parallel flow paths, down 
the water-cooled jackets, and up a single high-efficiency condenser exhausted to the atmosphere (Figure 6, center). The parallel flow paths were established to lower the internal pressure by raising the volume capacity of the flow path. A $3 \mathrm{~L}$ round bottom was put in place to collect the condensation of the evolved dissolution vapors. A lower drain spout allowed for easy removal of the volume collected. During the increased mass dissolution experiments (600 $\mathrm{g}$ instead of 300 $\mathrm{g}$ ), this vessel needed to be drained at least once during processing. The new chiller proved to be more efficient than the previous one, but was also unable to keep up with the exothermic process. On several occasions, the dissolution needed to be halted in order for the chiller to reestablish an acceptable chilled water supply.

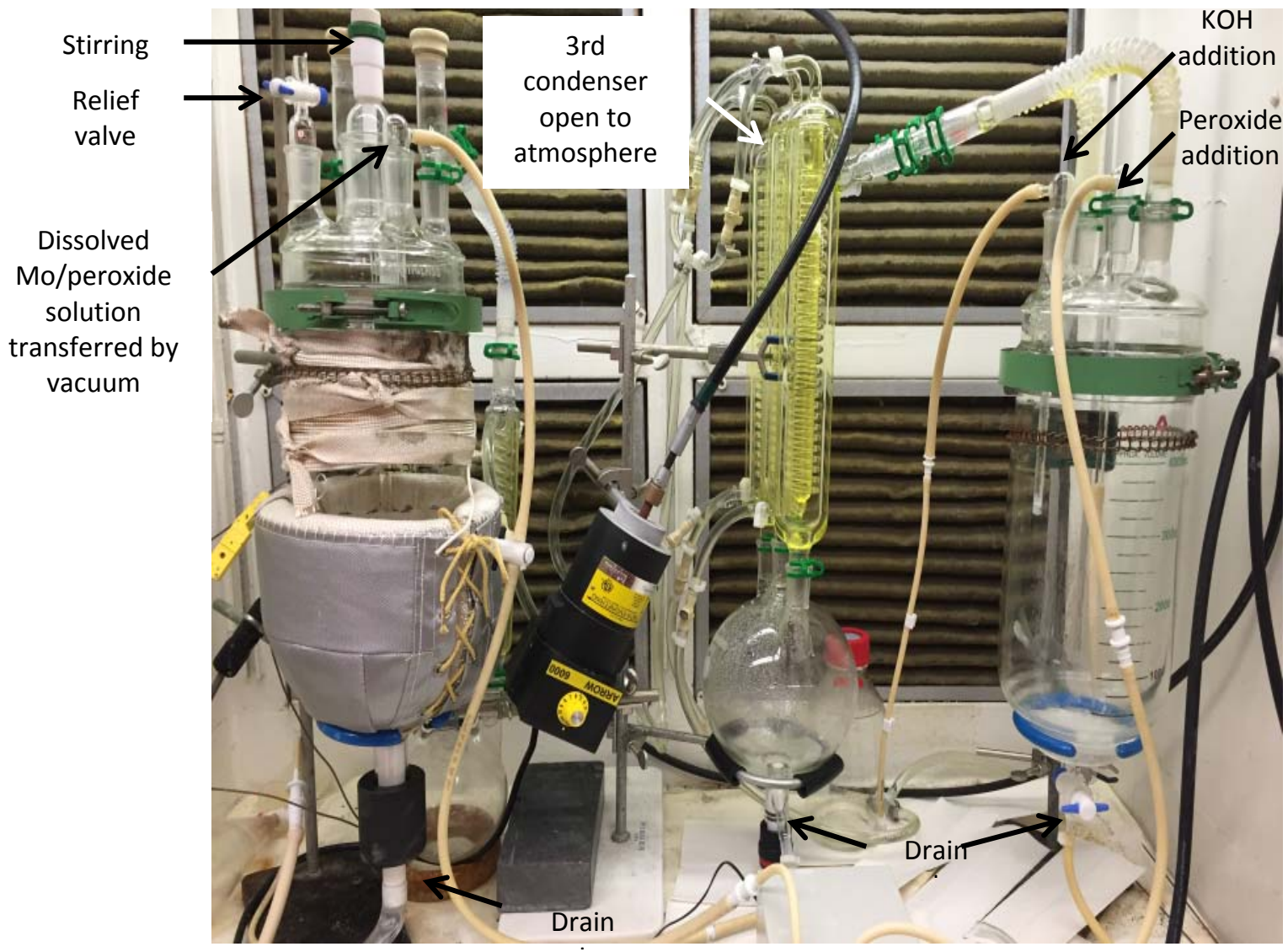

FIGURE 6 Dissolution apparatus for processing of up to $600 \mathrm{~g}$ of sintered Mo disks using $\mathrm{H}_{2} \mathrm{O}_{2}$. Evaporation vessel (left), is heated with an oversized heating mantel and heat tape. The associated condensation vessel is visible behind the evaporation vessel. The dissolution vessel (right) is connected to the evaporation vessel through the lower drain. The associated condensation vessel (center) is connected to the dissolution vessel by two flexible adapters and can be drained at any point during the experiment through a lower drain.

As the level of foam reached above half the height of the dissolution vessel, addition of $\mathrm{H}_{2} \mathrm{O}_{2}$ was slowed, and as the solution turned to a dark yellow-orange color, the dissolved portion of Mo was drained into the evaporation vessel. The dissolution was continued by adding fresh 
$\mathrm{H}_{2} \mathrm{O}_{2}$. This process was repeated throughout the dissolution of the Mo disks. In this manner, the dissolution and evaporation steps were performed in parallel, with partial solutions. Gentle heat $\left(50^{\circ} \mathrm{C}\right)$ was applied under vacuum to the initial volumes received in the evaporation/ concentration vessel to destroy excess $\mathrm{H}_{2} \mathrm{O}_{2}$ and begin the evaporation process. Because excessive heating causes rapid expulsion of gases and foam formation upon subsequent additions of dissolved Mo disks to the evaporation vessel, a relief valve was attached to relieve pressure and decrease the foam layer as needed.

After complete dissolution, the final portion of dissolved Mo was transferred to the evaporation vessel. The dissolution vessel was rinsed with KOH (Sigma Aldrich) (900 g of 85\% $\mathrm{KOH}$ dissolved in $740 \mathrm{~mL}$ of $\mathrm{H}_{2} \mathrm{O}$ ) in portions and then transferred to the evaporation vessel. Reaction of $\mathrm{KOH}$ with $\mathrm{H}_{2} \mathrm{O}_{2}$ is vigorous and this step was completed with extreme care. During evaporation, the solution was continuously stirred with an overhead stirrer (Arrow electric stirrer, CG-2021) using a glass shaft (CG-2074) with a Teflon paddle that was connected to the stirrer motor using a flexible drive shaft (CG-2047). The combined solutions were heated to destroy $\mathrm{H}_{2} \mathrm{O}_{2}$ and to bring the solution to the desired volume as required.

When the evaporation step was completed, the final solution was filtered by vacuum through a high-capacity filter (Polycap $36 \mathrm{TC}, 0.6 / 45 \mu \mathrm{m}$ ). Filtration was performed to remove impurities that precipitated under the alkaline conditions, such as iron. The filtration step usually required $\sim 15 \mathrm{~min}$. After filtration, $900 \mathrm{~g}$ of solid $85 \% \mathrm{KOH}$ was added. To make Mo $\sim 0.2 \mathrm{~g} / \mathrm{mL}$ as $\mathrm{K}_{2} \mathrm{MoO}_{4}$ in $5 \mathrm{M} \mathrm{KOH}$ solution, water was added as necessary to bring the density of the solution to $1.55 \mathrm{~g} / \mathrm{mL}$.

\section{Results and Discussion}

No significant changes were made to the evaporation vessel. The heating mantel, heat tape, and condensers performed as expected. Gently heating the solution that was transferred from the dissolution vessel accelerated the decomposition of $\mathrm{H}_{2} \mathrm{O}_{2}$. The solution, in general, was not heated to evaporation temperatures until after the complete dissolution of Mo and transfer into the dissolution vessel. If evaporation was required, the vessel was heated with both the heat tape and the heating mantel. Vacuum was applied at all times.

\section{Dissolution of $600 \mathrm{~g}$ of $26 \times 1 \mathrm{~mm}$ Disks}

The dissolution of $600 \mathrm{~g}$ of $26 \times 1 \mathrm{~mm}$ disks was not significantly different from the dissolution of $300 \mathrm{~g}$ disks [1]. The summary of dissolution tests with $600 \mathrm{~g}$ of sintered Mo disks is listed in Table 6. Dissolution rates were approximately the same from the optimized $300 \mathrm{~g}$ dissolution rates. With twice the mass of disks, approximately twice the amount of $\mathrm{H}_{2} \mathrm{O}_{2}$ and twice the amount of time was required to fully dissolve $600 \mathrm{~g}$ of disks. The slight increase in $\mathrm{H}_{2} \mathrm{O}_{2}$ can be attributed to the self-destruction of $\mathrm{H}_{2} \mathrm{O}_{2}$. Hydrogen peroxide decomposes with heat, and this reaction is vigorous and exothermic [2]. Consumption of $\mathrm{H}_{2} \mathrm{O}_{2}$ can be controlled by regulating the flow rate of $\mathrm{H}_{2} \mathrm{O}_{2}$ addition. The transfer of dissolved Mo to the evaporation vessel was determined by the color of the solution. As the color of the solution changed to dark yellow-orange, the solubility limit of the Mo-peroxo species was being reached; as a result, the 
flow of $\mathrm{H}_{2} \mathrm{O}_{2}$ was decreased or stopped and Mo-peroxo solution was drained to the evaporation vessel. This led to a more economical consumption of $\mathrm{H}_{2} \mathrm{O}_{2}$, which can shorten total processing times.

\section{Dissolution of $29.5 \times 0.5 \mathrm{~mm}$ Disks}

The dissolution rate of the larger-diameter and thinner disks was significantly faster than the $26 \times 1 \mathrm{~mm}$ disks (Table 6 ). The faster dissolution rate could partially be attributed to the larger surface area per mass of the disks. Assuming the porosities of the disks are equal, the surface area of the $29.5 \times 0.5 \mathrm{~mm}$ disks is $1,413.24 \mathrm{~mm}^{2}$, while the surface area of the $26 \times 1 \mathrm{~mm}$ disks is $1143.54 \mathrm{~mm}^{2}$. The increased surface area allows for greater contact with the $\mathrm{H}_{2} \mathrm{O}_{2}$ and thus more surface upon which the reaction may occur. Due to significantly better efficiency of dissolution, no additional time for evaporation was required, which led to significantly shorter total processing time.

TABLE 6 Results from optimization of large-scale dissolution experiments

\begin{tabular}{|c|c|c|c|c|c|c|c|}
\hline $\begin{array}{l}\text { Disks } \\
(\mathrm{mm})\end{array}$ & $\begin{array}{c}\text { Mass } \\
(\mathrm{g})\end{array}$ & $\begin{array}{c}\mathrm{H}_{2} \mathrm{O}_{2} \\
(\mathrm{~L}) \\
\end{array}$ & $\begin{array}{c}\text { Water } \\
\text { Condensed } \\
\text { from Diss. } \\
(\mathrm{L}) \\
\end{array}$ & $\begin{array}{c}\text { Diss. } \\
\text { Time } \\
(\mathrm{hr})\end{array}$ & $\begin{array}{c}\text { Evaporation } \\
\text { Time } \\
\text { (hr) }\end{array}$ & $\begin{array}{c}\text { Total } \\
\text { Time } \\
\text { (hr) } \\
\end{array}$ & $\begin{array}{c}\text { Total } \\
\text { Diss. } \\
\text { Rate } \\
\text { (g/min) }\end{array}$ \\
\hline $26 \times 1$ & 603.7 & 9.5 & 5.4 & 2.5 & 1.5 & 4.5 & 2.24 \\
\hline $26 \times 1$ & 600.5 & 9.0 & 5.9 & 2.75 & 1 & 4.0 & 2.5 \\
\hline $26 \times 1$ & 602.4 & 6.5 & 4.3 & 2.0 & 1.5 & 3.75 & 2.68 \\
\hline $29.5 \times 0.5$ & 596.8 & 6.8 & 4.7 & 1.8 & 0 & 2.25 & 4.42 \\
\hline
\end{tabular}

\section{Reaction Heat and Cooling Capacity}

During the last dissolution of $26 \times 1 \mathrm{~mm}$ disks, thermocouples were installed to monitor changes in the temperature at four different locations (Figure 7). Temperatures were monitored at:

$\mathrm{T}_{1}$ - inlet of coolant before entering the first condenser

$\mathrm{T}_{2}$ - outlet of coolant after third condenser (directly connected to dissolution vessel)

$\mathrm{T}_{3}$ - water vapor on top of the dissolution vessel

$\mathrm{T}_{4}$ - water vapor exiting the last condenser (open to atmosphere) 

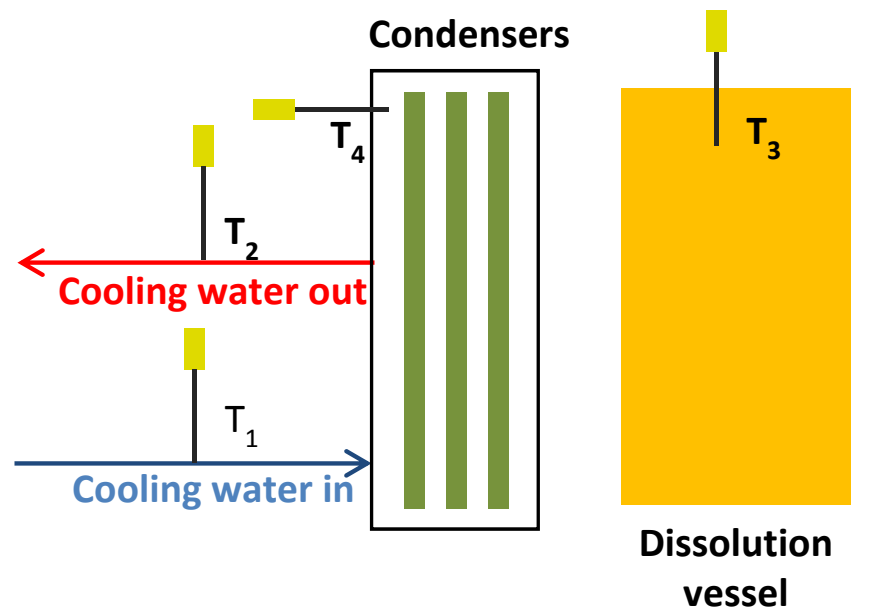

FIGURE 7 Schematic of dissolution setup with placement of thermocouples to monitor temperatures during dissolution of $600 \mathrm{~g}$ batch of sintered Mo disks

The temperature profiles of the four points monitored are shown in Figure 8 . The headspace temperature of the dissolution vessel rose from $24^{\circ} \mathrm{C}$ to $99^{\circ} \mathrm{C}$ within $30 \mathrm{sec}$. Due to the heat of reaction, the temperature of coolant leaving the condensers rose from $-0.3^{\circ} \mathrm{C}$ to $85^{\circ} \mathrm{C}$ in $\sim 15$ min of processing time. As dissolution began, gas was observed exhausting from the last condenser (open to atmosphere). However, the temperature of those gases decreased from $6^{\circ} \mathrm{C}$ to $-2^{\circ} \mathrm{C}$ in first $30 \mathrm{sec}$ of dissolution. This suggests that at this point of dissolution, exhausted gas is oxygen and not water vapor. However, as the dissolution progressed the temperature began to increase, reaching $\sim 23^{\circ} \mathrm{C}$ after $\sim 14 \mathrm{~min}$. During the $2 \mathrm{hr}$ of dissolution, it was observed that the cooling capacity of the chiller was not sufficient and the temperature at the exhaust point of the condenser open to atmosphere reached $60^{\circ} \mathrm{C}$ (not shown in Figure 8), indicating that some portion of water vapor may have been exhausted. However, the amount of water vapor that escaped was minimal, as there were only short periods of time with elevated exhaust temperatures. 


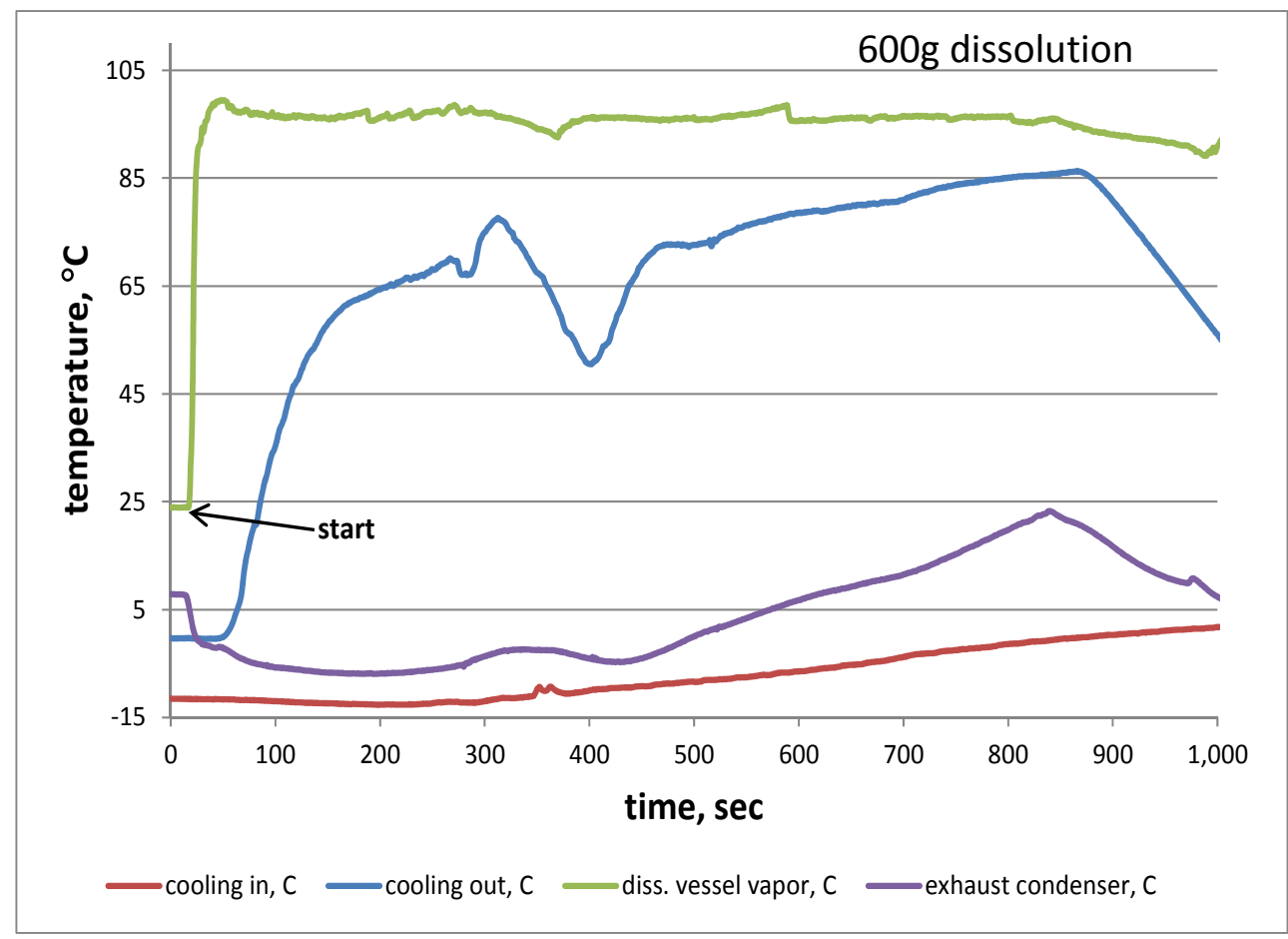

FIGURE 8 Temperature profiles during first $15 \mathrm{~min}$ for dissolution of $600 \mathrm{~g}$ of sintered Mo disks in $50 \% \mathrm{H}_{2} \mathrm{O}_{2}$

Thermal images of the system before and during dissolution are shown in Figure 9. Figures $9 \mathrm{a}$ and $9 \mathrm{~b}$ show the thermal images of dissolution setup before dissolution, while Figures $9 \mathrm{c}-9 \mathrm{~h}$ are thermal images from during the dissolution. The volume of water condensed in the first 15 min of dissolution was used to determine the heat of dissolution:

$$
\Delta \mathrm{H}=\operatorname{mc} \Delta \mathrm{T}
$$

where $\Delta \mathrm{H}$ is the heat absorbed by water, $\mathrm{m}$ is mass, and $\mathrm{c}$ is specific heat $\left(\mathrm{c}=4.18 \mathrm{~J} / \mathrm{g} /{ }^{\circ} \mathrm{C}\right)$. The mass of water that condensed during the first 15 min of the dissolution was $\sim 900 \mathrm{~g}$; the difference between room temperature and the boiling temperature of water was calculated as $\Delta \mathrm{T}$ $=76^{\circ} \mathrm{C}$. 


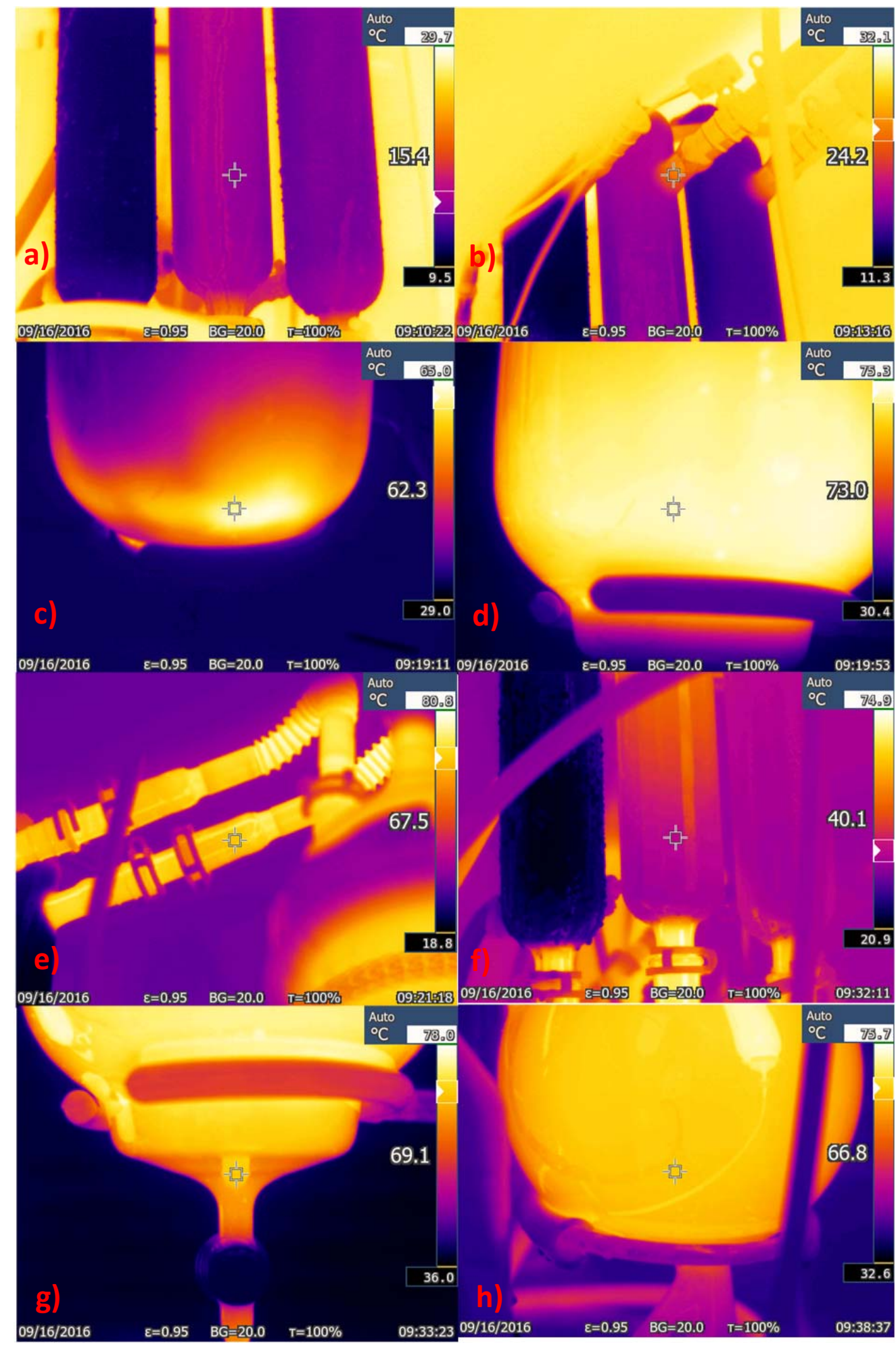

FIGURE 9 Thermal images of: (a, b) condensers before dissolution started; (c, d) dissolution vessel during dissolution; (e) flexible adapters between dissolution vessel and condensers; (f) condensers during dissolution; (g) bottom of dissolution vessel during dissolution; (h) condensation flask containing condensed water from dissolution 
$\Delta \mathrm{H}$ determined based on Eq. 1 was $286.3 \mathrm{~kJ} . \Delta \mathrm{H}_{\text {vap }}=2,257 \mathrm{~kJ} / \mathrm{kg}$ of water; therefore, total heat from reaction was:

$$
\Delta \mathrm{H}_{\mathrm{T}}=\Delta \mathrm{H}+0.9 \Delta \mathrm{H}_{\mathrm{vap}}=2,317.6 \mathrm{~kJ}
$$

where 0.9 represents $0.9 \mathrm{~kg}$ of water $(900 \mathrm{~mL})$. This was converted to power with Eq. 3:

$$
\mathrm{P}=\Delta \mathrm{H}_{\mathrm{T}} / \mathrm{t}=2,317.6 / 900=2.58 \mathrm{~kW}
$$

where $t$ is time. Based on these calculations, the capacity of the chiller used in this experiment (see Experimental section) was far exceeded. A chiller with higher cooling capacity should be used in the future if release of water is of concern. In our experimental setup, water vapor was exhausted inside of a chemical fume hood and did not cause concern; however, release of water vapor in hot cell can cause significant fogging and pooling or free-standing water. An example of a chiller with sufficient cooling capacity is the Julabo FL4003 recirculating cooler, which has $4 \mathrm{~kW}$ cooling capacity at $20^{\circ} \mathrm{C}$, and $2.4 \mathrm{~kW}$ at $0^{\circ} \mathrm{C}$.

\section{Summary and Conclusions}

Dissolution studies were performed on $600 \mathrm{~g}$ of Mo disks with an optimized system, developed from multiple $300 \mathrm{~g}$ dissolution studies. The average dissolution time from the three experiments performed with $26 \times 1 \mathrm{~mm}$ disks was $\sim 4.1 \mathrm{hr}$, giving average dissolution rates of $2.3 \mathrm{~g} / \mathrm{min}$. These large-batch rates were faster than those observed for single $26 \times 1 \mathrm{~mm}$ disks. Shorter total processing time was observed during the processing of $29.5 \times 0.5 \mathrm{~mm}$ disks because the additional time for evaporation was not required. When the optimized system was used, the dissolution rate for $600 \mathrm{~g}$ of thinner disks was $4.44 \mathrm{~g} / \mathrm{min}$, which was significantly faster than for the thicker $26 \times 1 \mathrm{~mm}$ disks. The increased condenser capacity helped to minimize the amount of vapor to escape the system, conservatively estimated to be $<5 \%$. To eliminate all vapor escape, a recirculating chiller with $>3 \mathrm{~kW}$ cooling capacity is required. 


\section{ADAPTATION OF BENCHTOP OPTIMIZED DISSOLUTION SYSTEM TO HOT CELL OPERATIONS}

Processing production-level quantities — thousands of curies — of irradiated Mo will be performed in a hot cell. NorthStar is currently designing its hot cell for this work. One of the primary concerns is that the system described in Section 2 of this report is composed entirely of glass. Glass could break within a hot cell, where metallic manipulators perform the operations needed for processing hundreds of grams of irradiated Mo. Furthermore, the system requires cleaning between processing runs, and periodic maintenance. Therefore, there is a need to demonstrate that the equipment can be set up, maintained, and operated in a hot cell environment. Argonne has configured a hot cell mockup to determine the required dimensions for the optimized system and ensure operations can be performed within the confined space of a hot cell.

Figure 10 shows an image of the hot cell mockup (sketches of modules can be found in Appendix B). The system fits within the dimensions of $140 \times 112 \times 160 \mathrm{~cm}(\mathrm{w} \times \mathrm{d} \times \mathrm{h})$. This layout allows for additional $\sim 70 \mathrm{~cm}$ of working space in front of the setup. These dimensions allow ample room for an antechamber tray pass-through, additional valves, and other required attachments (e.g., condenser water lines, liquid disposal lines). There is also room for retrieving disks from irradiation holders, preparing disks to add to the dissolution vessel, performing standard operations, and storing the manipulators when not in use. The manipulators must be able to reach from floor to the height of the tallest vessel $(0-127 \mathrm{~cm})$. The system components are modular so that individual components can be removed and replaced when maintenance or replacement is needed.

Because they are too heavy and cumbersome to remove with manipulators alone, the modules are equipped with rings on the top to allow hooks from cranes to lift them. The manifolds are made of lightweight aluminum and can be bolted to the floor to avoid inadvertently knocking them over. Alternatively, the bases could be made to fit into notches in the hot cell floor or a lock-and-key mechanism could be devised. A large exterior hot cell door would be required to bring the modules in and out of the hot cell, or a long antechamber in which the modules could lay on their sides could be devised.

Flexible adapters are used to connect the evaporation vessel to its condenser system and the dissolution vessel to its condenser system. These flexible adapters are required unless fixed positioning is used for the system components. Even then, the system may prove difficult to wield with manipulators. Every connection must be gas tight, to provide adequate vacuum and no loss of material or vapor. It is unclear at this point whether flexible or rigid connections better suit the system. 


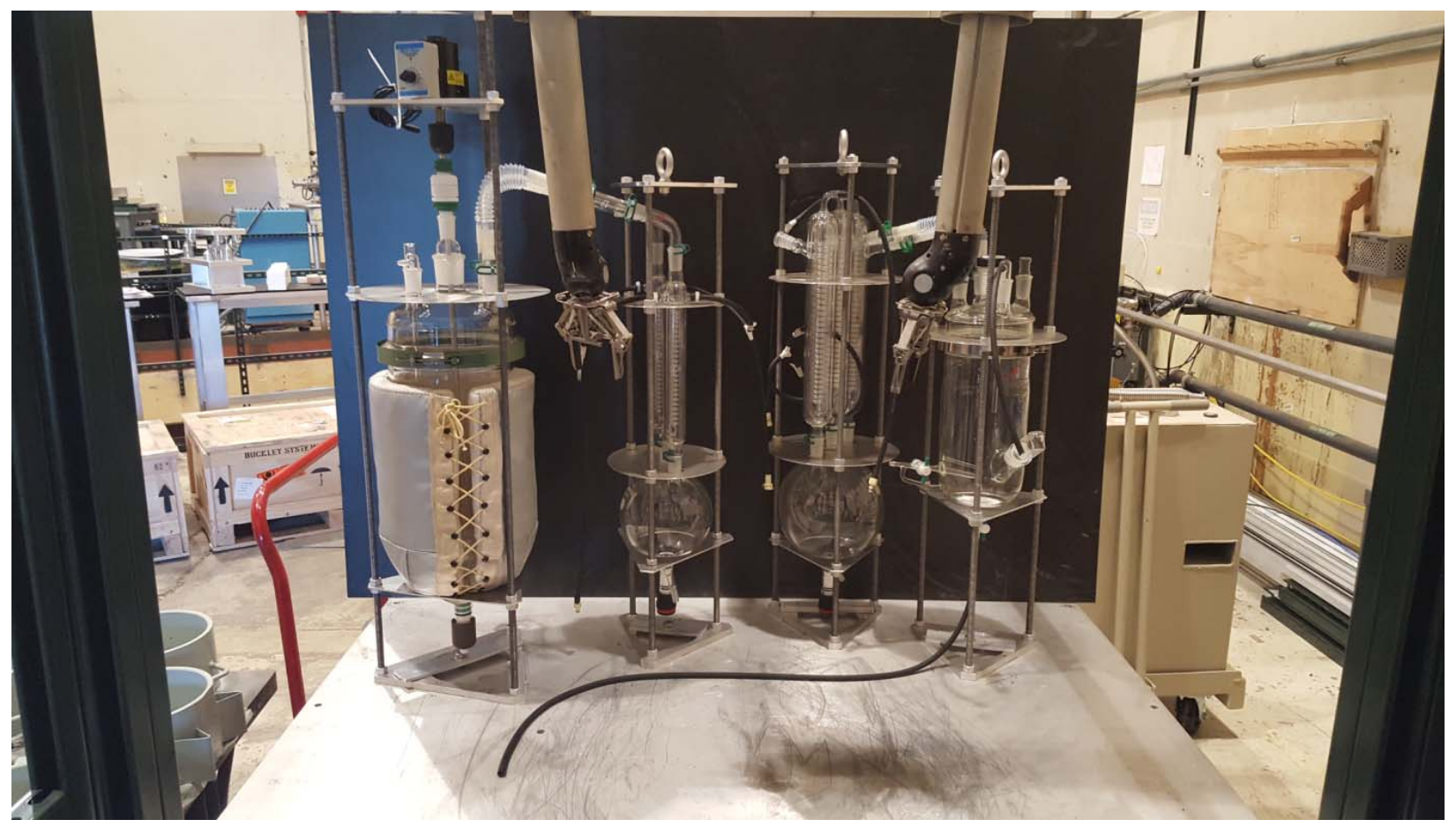

FIGURE 10 Hot cell mockup with view from operator standpoint. No leaded glass is in place. Left to right: evaporation vessel, evaporation vessel condenser and collection vessel, dissolution vessel condensers and collection vessel, and dissolution vessel.

\section{Evaporation System}

The evaporation vessel (Figures 11A and 11B) is currently $\sim 127 \mathrm{~cm}$ in height, with a diameter of $\sim 34 \mathrm{~cm}$ at its widest point. The associated collection system (Figure 11C) has a height of $\sim 97 \mathrm{~cm}$ and a diameter of $\sim 24 \mathrm{~cm}$ at its widest point. A glass evaporation vessel is needed to view the interior liquid level, as the vessel is too cumbersome to weigh. Graduations can be marked on the exterior of the vessel to indicate the solution volume. The vessel alone is $\sim 85 \mathrm{~cm}$ tall. The vessel's head, shown in Figure 11B, adds another $\sim 35 \mathrm{~cm}$ from the combined $\sim 18 \mathrm{~cm}$ head and the $\sim 17 \mathrm{~cm}$ adapter to the stirring mechanism. The condenser system is attached with flexible adapters with glass 24/40 (standard taper) adapters.

The condenser system connects to the evaporation vessel by two pathways, to maximize the flow path volume. Two high-efficiency condensers and a gas inlet are affixed to a 3 L roundbottom vessel with a bottom drain. The bottom drain is currently controlled with a twist stopcock that can be replaced with a T-valve (for use with manipulators) or with a remotely operable valve. Condensate must be drained after every process. 

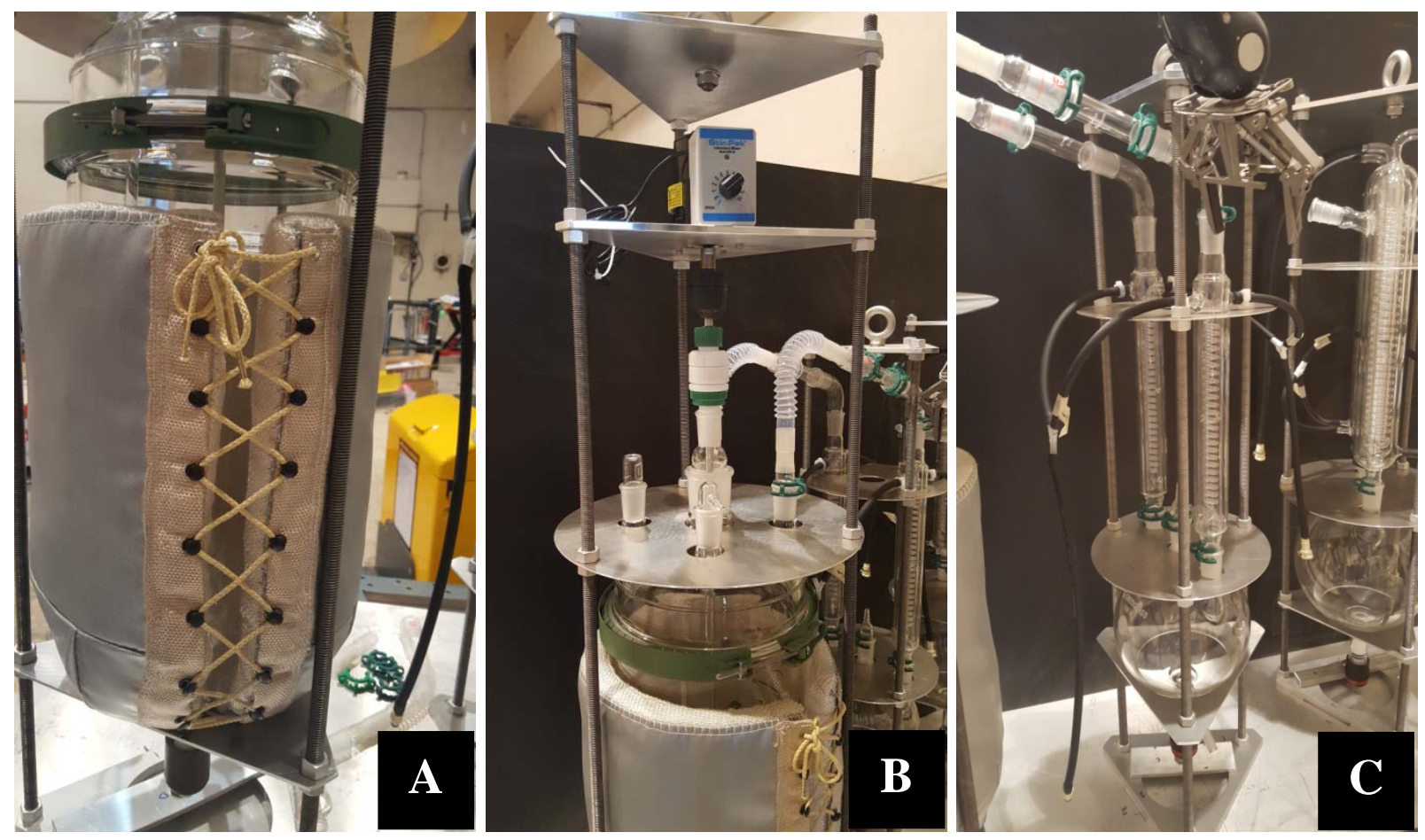

FIGURE 11 (A) Evaporation vessel with heating mantel on 10L reaction vessel; (B) Evaporation vessel head with associated stirrer and connections to condenser system; and (C) Evaporation condenser system.

\section{Dissolution System}

The dissolution vessel (Figures $12 \mathrm{~A}$ and $12 \mathrm{~B}$ ) has a height of $\sim 97 \mathrm{~cm}$ and a diameter of $\sim 31 \mathrm{~cm}$ at its widest point. Figure 12C shows the associated condenser system; it has a height of $\sim 102 \mathrm{~cm}$ and a diameter of $\sim 28 \mathrm{~cm}$ at its widest point. The glass vessel is needed to view the dissolution process. The operator must be able to observe the disks, the color of the solution, and the height of the reaction foam. The vessel itself is $\sim 66 \mathrm{~cm}$ tall. The vessel's head is $\sim 16.5 \mathrm{~cm}$ tall and the flexible adapters add another $\sim 5 \mathrm{~cm}$. The condenser system is attached with flexible adapters with glass 24/40 (standard taper) adapters.

The condenser system connects to the dissolution vessel by two pathways, to maximize the flow path volume. Three high-efficiency condensers are affixed to a $5 \mathrm{~L}$ round-bottom vessel with a bottom drain. The bottom drain is currently controlled with a twist stopcock that can be replaced with a T-valve (for use with manipulators) or with a remotely operable valve.

Condensate must be drained after every process. The central condenser is open to the atmosphere to avoid pressurization of the system. 

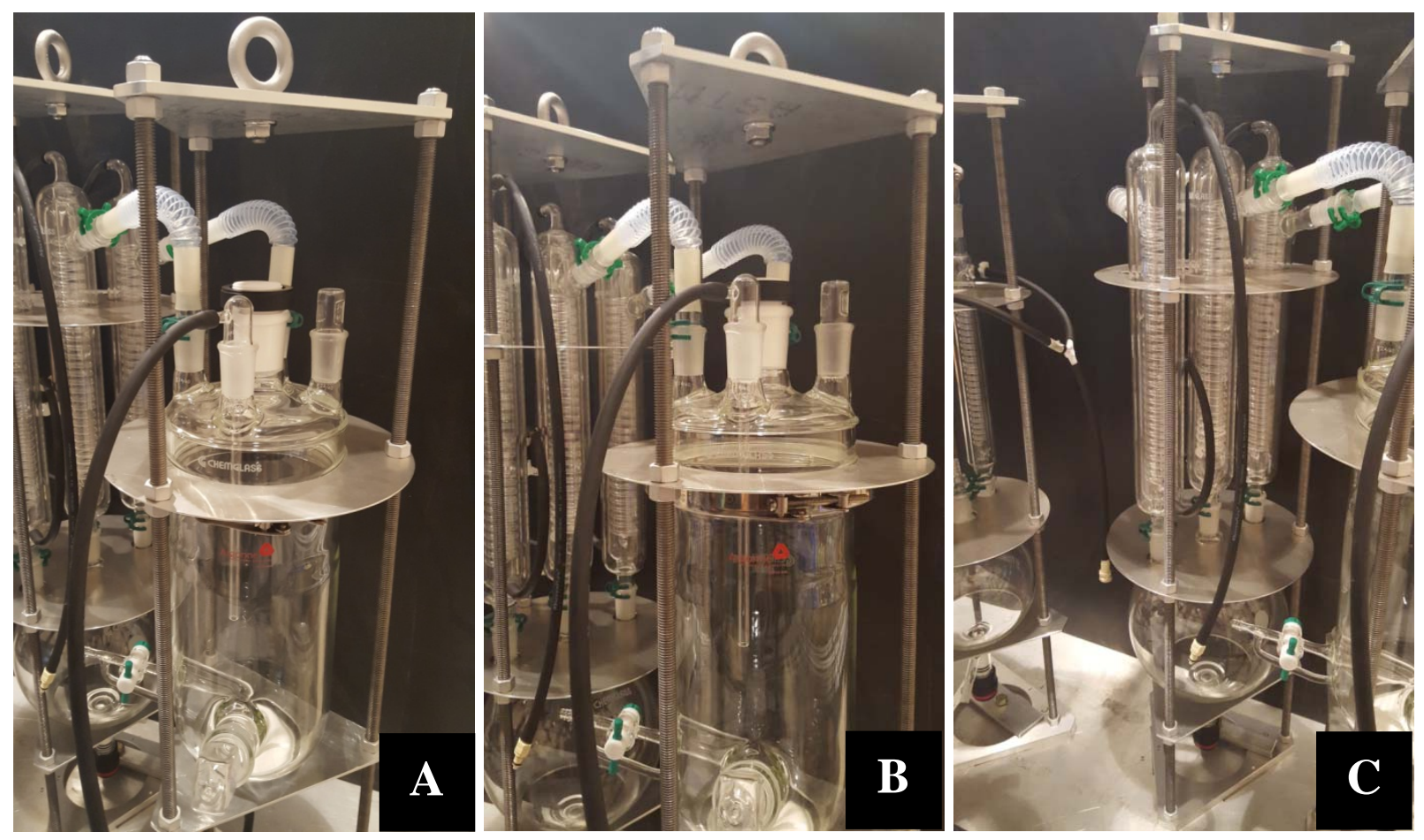

FIGURE 12 Dissolution vessel (A and B) and dissolution vessel condenser system (C)

\section{Conversion to Stainless Steel}

Stainless steel provides a chemically resistant material with durability that far exceeds glass. As previously mentioned, the dissolution and evaporation vessels must remain glass. A shield or shroud can be used to protect the vessels from inadvertent bumps with manipulators or other tools within the hot cell. Components eligible for conversion to stainless steel are the condensation systems (dissolution and evaporation), including the evaporation and dissolution vessel heads/lids. The vessel heads can be replaced with stainless steel flat caps to eliminate some of the headspace needed.

Introducing the disks to process through the flat cap of the dissolution vessel can be accomplished by several methods. One is to include a slide mechanism, where the disks are loaded one at a time. Another, more efficient, method is to use an insert that is loaded with the disks (presumably the irradiation target holder), introduce the disks into the dissolution vessel through a pass-through in the stainless steel cap, and have a mechanism that releases the disks into the vessel. The pass-through can be sealed with a plug once the target holder releases its payload. This requires a modest redesign of the dissolution holder. Flexible or rigid connections can be used to connect the modular systems together. Aside from the durability, an added benefit to condensation systems of stainless steel is that remotely operated or electric valves can be used to drain the system, minimizing remote operator manipulations. 


\section{ACKNOWLEDGMENTS}

Work supported by the U.S. Department of Energy's National Nuclear Security Administration, Office of Material Management and Minimization, under Contract DE-AC0206CH11357. Argonne National Laboratory is operated for the U.S. Department of Energy by UChicago Argonne, LLC.

\section{REFERENCES}

1. P. Tkac, D.A. Rotsch, D Stepinski, V. Makarashvili, G.F. Vandegrift, J. Harvey, Optimization of the Processing of Mo Disks, Argonne National Laboratory, Report ANL/NE-15/46 (January 2016).

2. P. Tkac, G. Vandegrift, J. Harvey, Dissolution of Sintered Mo Disks, Argonne National Laboratory, Report ANL/CSE-13/19 (July 2012).

3. P. Tkac, G. Vandegrift, S.D. Nunn, and J. Harvey, Processing of Sintered Mo disks Using Hydrogen Peroxide, Argonne National Laboratory, Report ANL/CSE-13/44 (September 2013).

4. P. Tkac, G.F. Vandegrift, Dissolution of Sintered Mo Disks, Argonne National Laboratory, Report ANL/CSE-14/26 (September 2014).

5. P. Tkac, G.F. Vandegrift, Recycle of Enriched Mo Targets for Economic Production of ${ }^{99} \mathrm{Mo} /{ }^{99 \mathrm{~m}} \mathrm{Tc}$ Medical Isotope without Use of Enriched Uranium, J. Rad. Nucl. Chem. 308: 205-212 (2016).

6. P. Tkac, M.A. brown, S. Sen, D. Bowers, K. Wardle, J.M. Copple, K. Pupek, T.L. Dzwiniel, C. Pereira, G.K. Krumdick, G.F. Vandegrift, Development of Solvent Extraction Approach to Recycle Enriched Molybdenum Material, Argonne National Laboratory, Report ANL/NE-16/8 (2016).

7. R.A. Lowden, S.D. Nunn, J.O. Kiggans Jr., R.J. Parten, Bryan C.D. Powder Metallurgy Fabrication of Molybdenum Accelerator Target Disks, Oak Ridge National Laboratory, Report ORNL/TM-2014/238 (July 2015). 


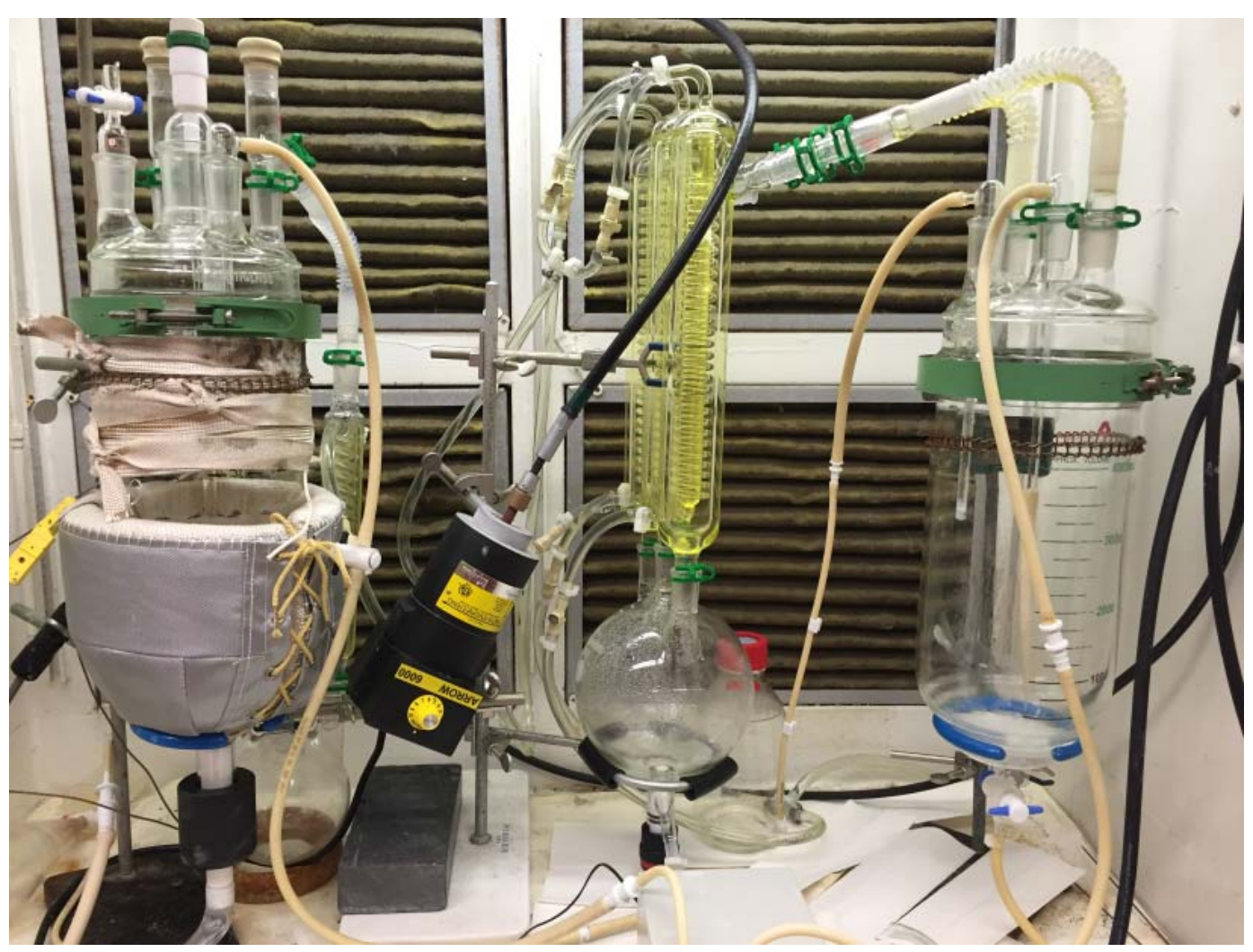

FIGURE A.1 Custom-made dissolution vessel (modified from $4 \mathrm{~L}$ reaction vessel, ChemGlass CG-1920-05)

Dissolution vessel lid 4-neck, 24/40 joint - ChemGlass CG-1941-03 www.chemglass.com/product_view.asp?pnr=CG-1941

Flexible adapters male to female 24/40 - SigmaAldrich ST/NS 24/40 - Z530891 www.sigmaaldrich.com/catalog/product/aldrich/z530891?lang=en\&region=US

4L evaporation vessel with drain valve - ChemGlass CG-1924-05 www.chemglass.com/product_view.asp?pnr=CG-1924

High Efficiency condensers for diss. vessel. (3pcs) - ChemGlass CG-1215-C-01 www.chemglass.com/product_view.asp?pnr=CG-1215-C

3L condensing flask 3-neck with drain valve - ChemGlass CG-1523-V-04 www.chemglass.com/product_view.asp?pnr=CG-1523-V

Evap. Vessel lid - 5 neck, 24/40 - ChemGlass CG-1946-01 
www.chemglass.com/product_view.asp?pnr=CG-1946

Stirrer bearing - ChemGlass CG-2077-U-01

www.chemglass.com/product_view.asp?pnr=CG-2077-U

Stirrer shaft - ChemGlass CG-2074-01

www.chemglass.com/product_view.asp?pnr=CG-2074

Arrow overhead stirrer - ChemGlass CG-2021-01

www.chemglass.com/product_view.asp?pnr=CG-2021

Flexible drive shaft - ChemGlass CG-2047-01

www.chemglass.com/product_view.asp?pnr=CG-2047

Clamp for reaction flanges (2pcs) - ChemGlass CG-141-T-14

www.chemglass.com/product_view.asp?pnr=CG-141-T

Heating mantle for $4 \mathrm{~L}$ vessel with drain - ChemGlass CG-10009-18

www.chemglass.com/product_view.asp?pnr=CG-10009

Condenser for evaporation vessel (2pcs) - ChemGlass CG-1215-A-10

www.chemglass.com/product_view.asp?pnr=CG-1215-A

1L condensing flask 3-neck with drain valve - ChemGlass CG-1523-V-02

www.chemglass.com/product_view.asp?pnr=CG-1523-V

Adapters $75^{\circ}$ angle (2pcs) - 24/40 joint ChemGlass CG-1022-01

www.chemglass.com/product_view.asp?pnr=CG-1022

Adapter straight (2pcs) - 24/40 joint ChemGlass CG-1003-01

www.chemglass.com/product_view.asp?pnr=CG-1003

Inlet adapter 24/40joint (3pcs) ChemGlass CG-1014-E

www.chemglass.com/product_view.asp?pnr=CG-1014-E

Flow control adapter - ChemGlass CG-1029-G-01

www.chemglass.com/product_view.asp?pnr=CG-1029-G

JKEM controller MODEL 270

www.jkem.com/high-safety-controller-model-270

Recirculating cooler - Julabo FL 1701 (recommend one with higher cooling capacity; this one had highest capacity with 110V)

https://punchout.fishersci.com/shop/products/f11701-cooler-20-40c/507533950

www.julabo.com/us/products/recirculating-coolers/f11701-recirculating-cooler

Vacuum pump - plenty of options, should be corrosion resistant 


\section{APPENDIX B: HAND SKETCHES FOR HOT CELL MOCKUP HOLDERS}
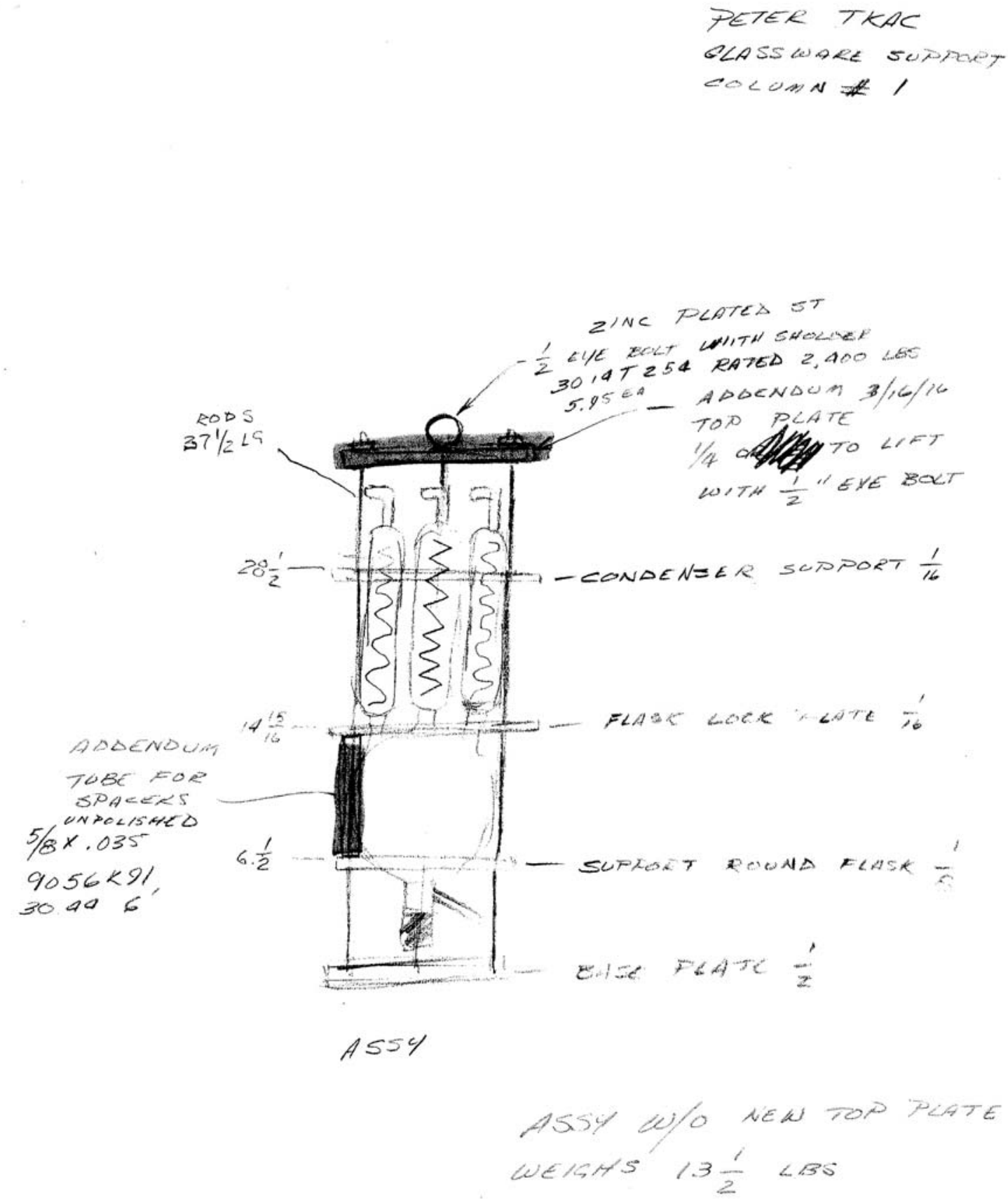
$1 / 3$

PEOCDANS 1005

108113 OUTSIDE CONTOUR

108113 , CENTEE NOLE

$1081132 \frac{1}{2}-13$ HOLES

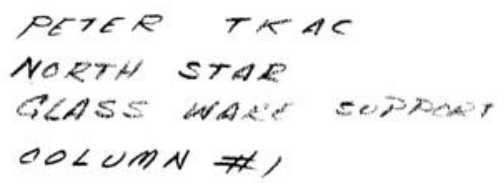

$R 5 \frac{3}{8}$
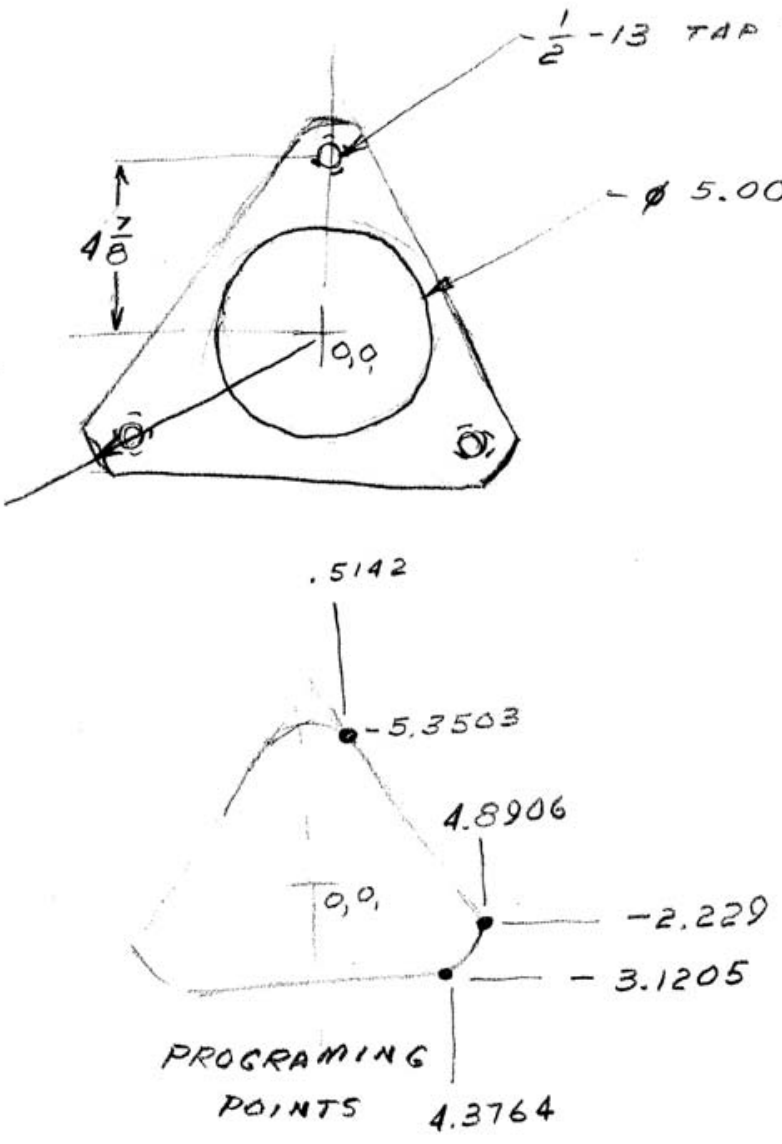

BASE PLATE $1 / 2$ "ALUMNUN STK 


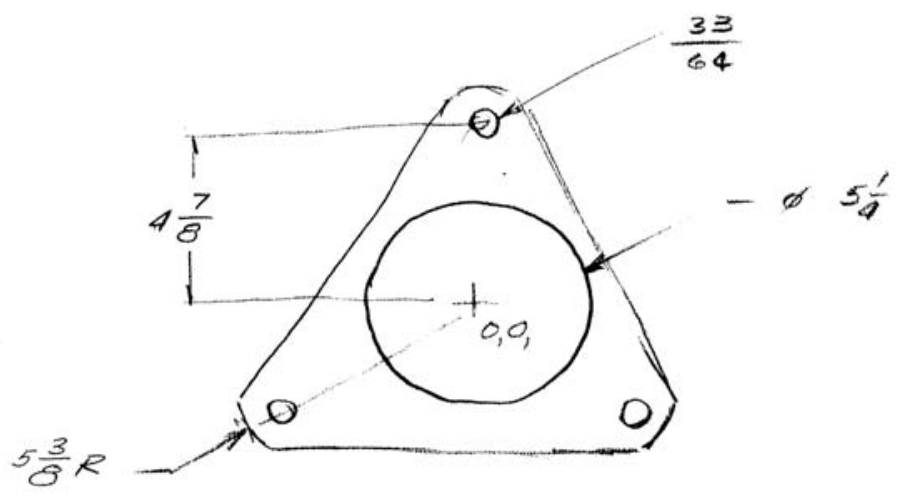

$$
\begin{aligned}
& \text { FIRST PLATE WD } \\
& \text { FROM EASE } \frac{1}{\mathrm{~S}} \text { ALUNINUN } \\
& \text { SLPPOETS ROUND FLASK }
\end{aligned}
$$

Hoces

1061133

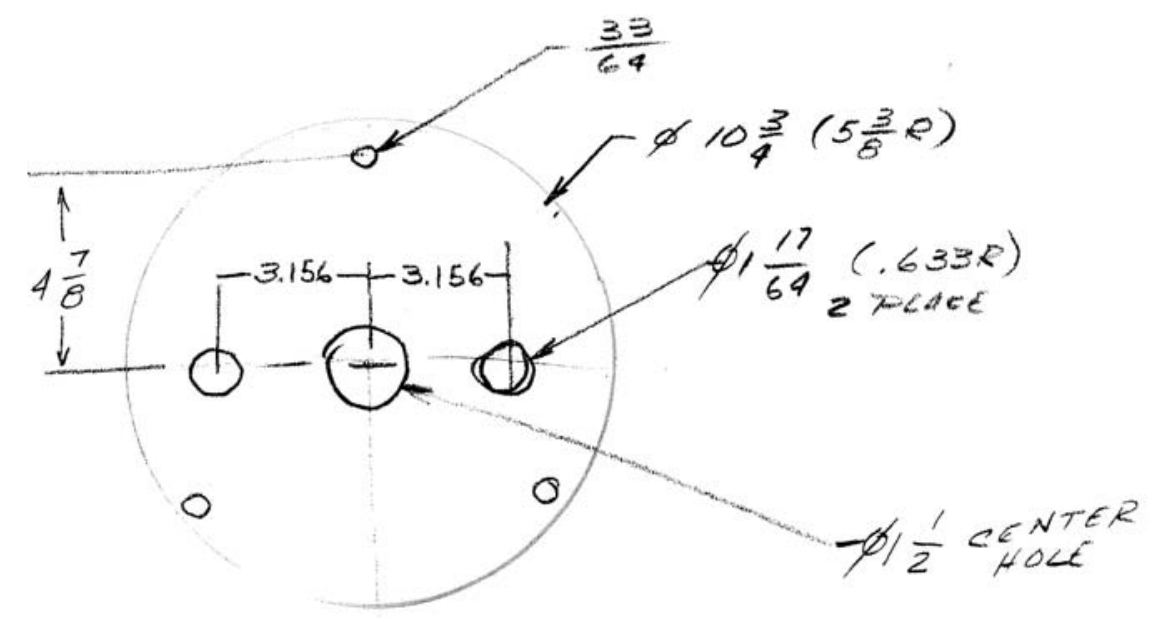

$$
\begin{aligned}
& 2 \text { ND PLATE UP, TOP } \\
& \text { OF ROUNA FLASE } \frac{1}{16} \text { ALUMNOM }
\end{aligned}
$$


$3 / 3$

DETEE TKAC NORTA STAR CLASSwa.e support
COLUMN \#।

10811340.0.

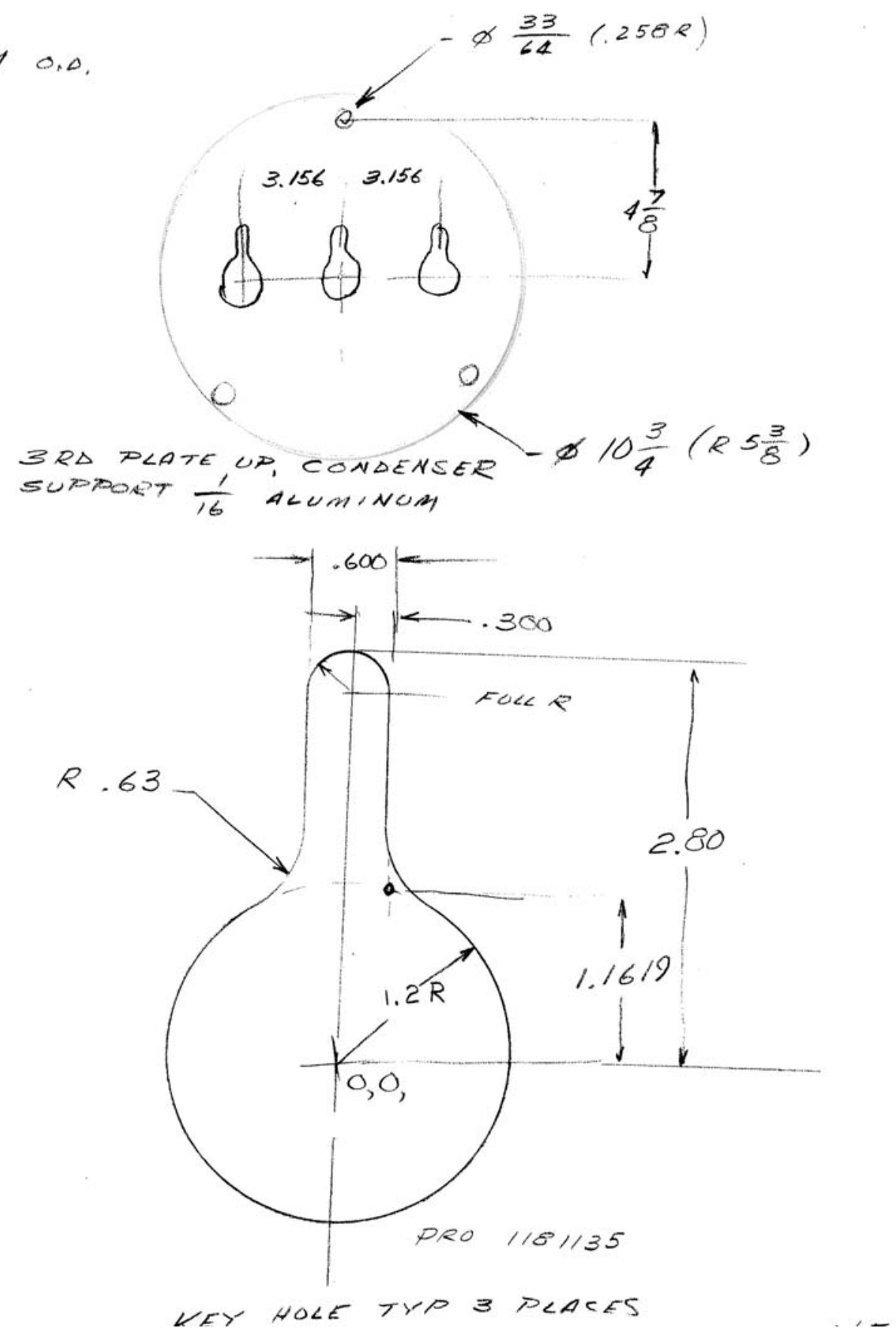

29 


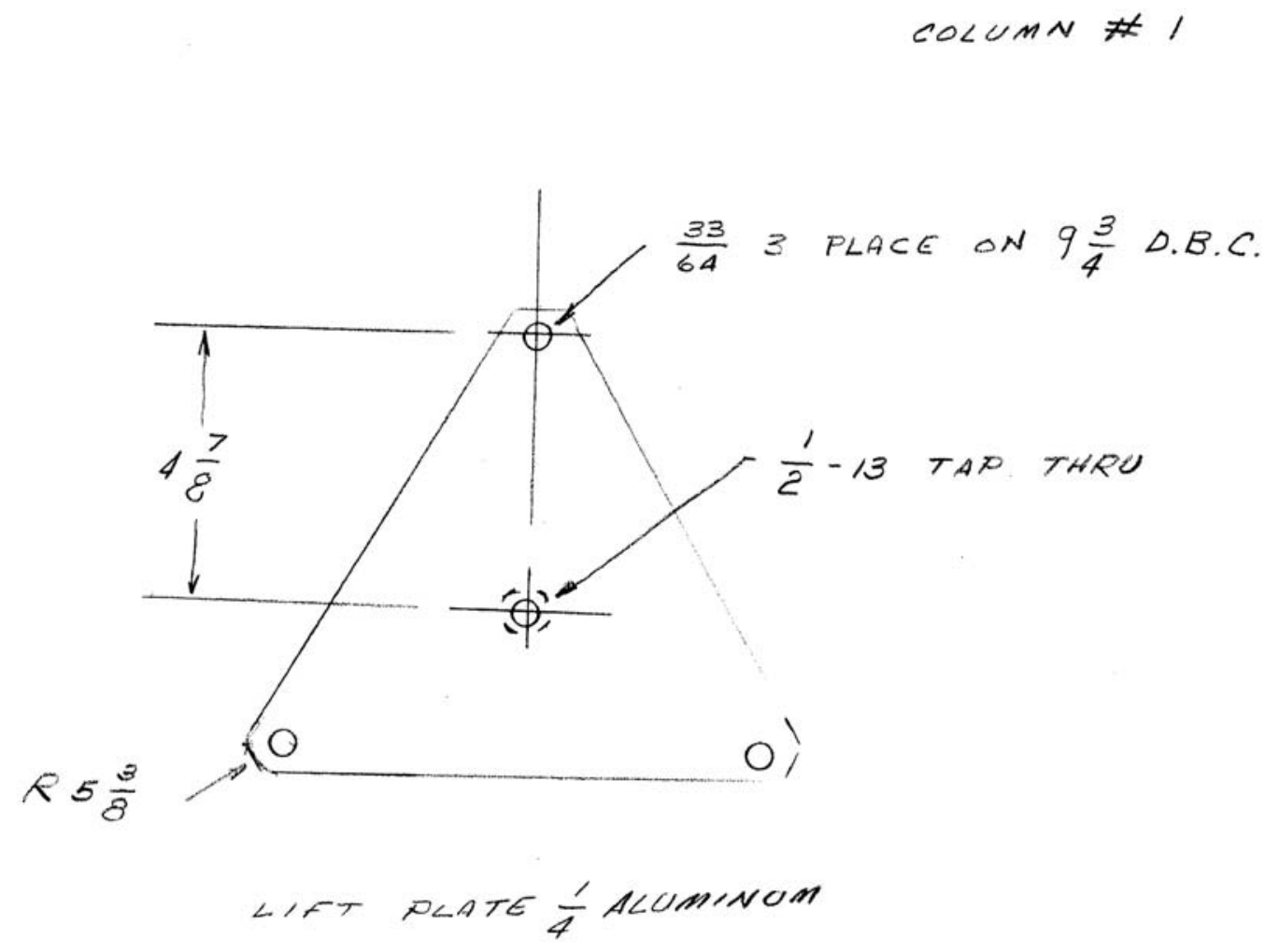

$5 / 5$ 


$$
\begin{gathered}
\text { colunN } \\
455 \%
\end{gathered}
$$

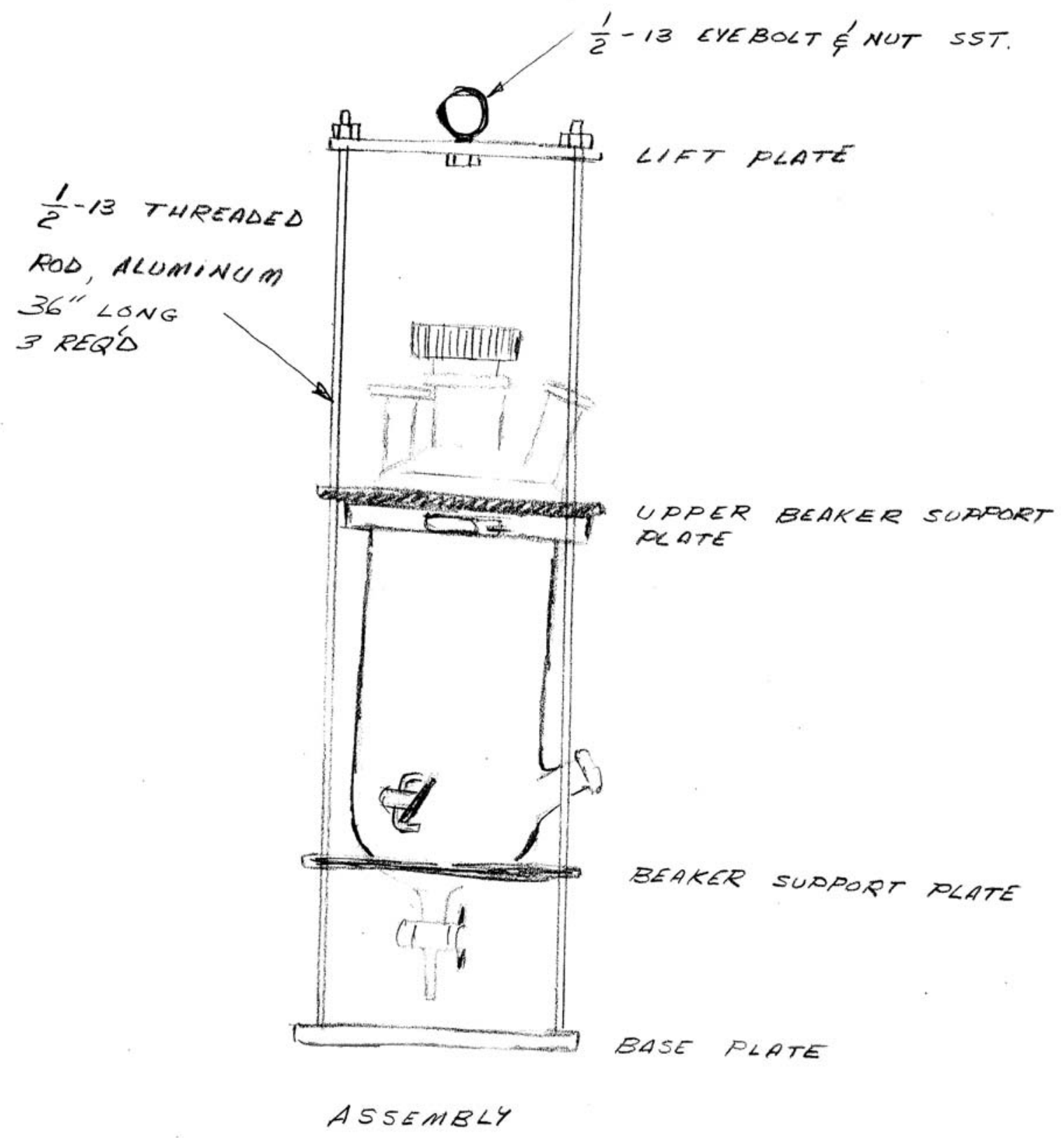




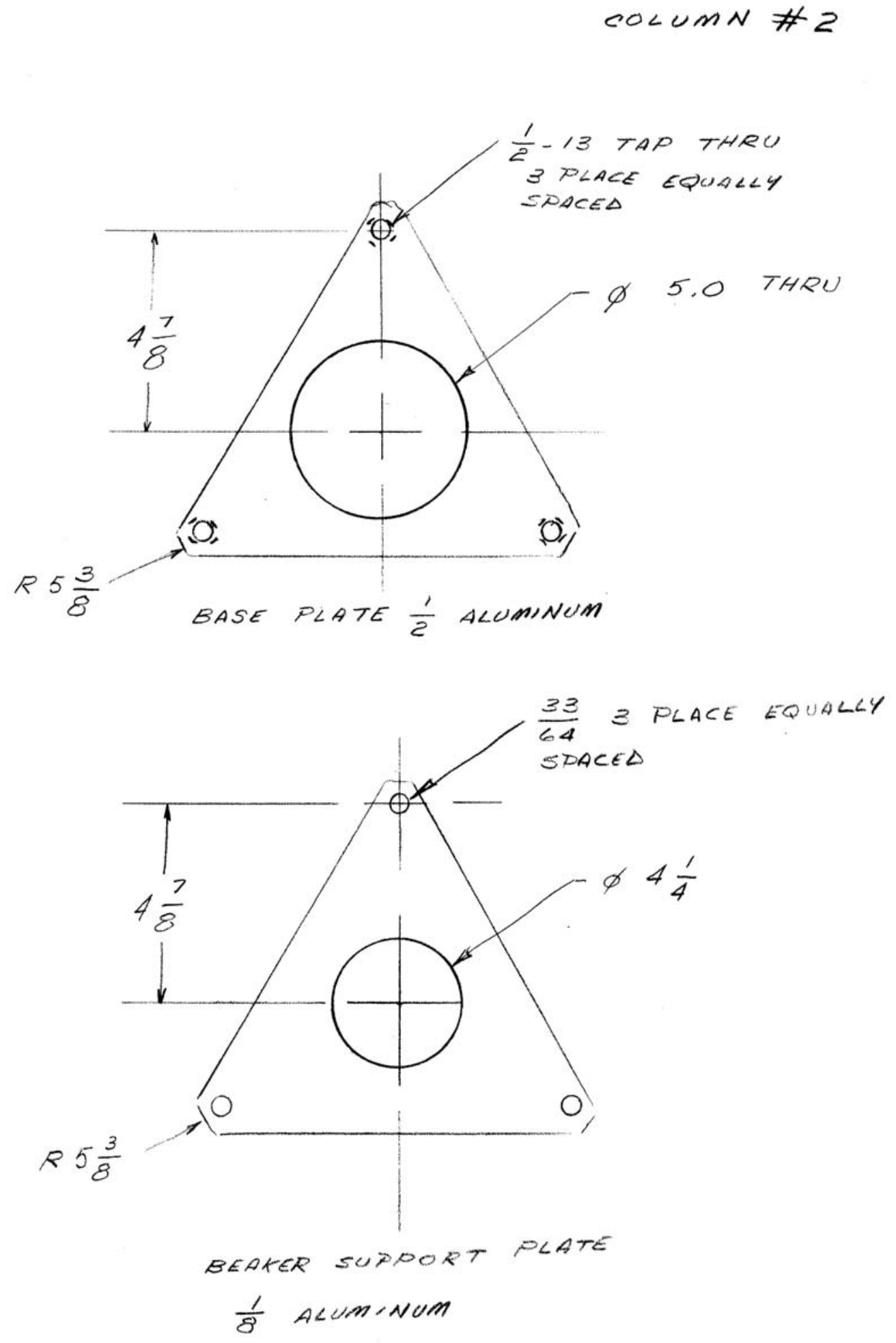

$2 / 2$ 


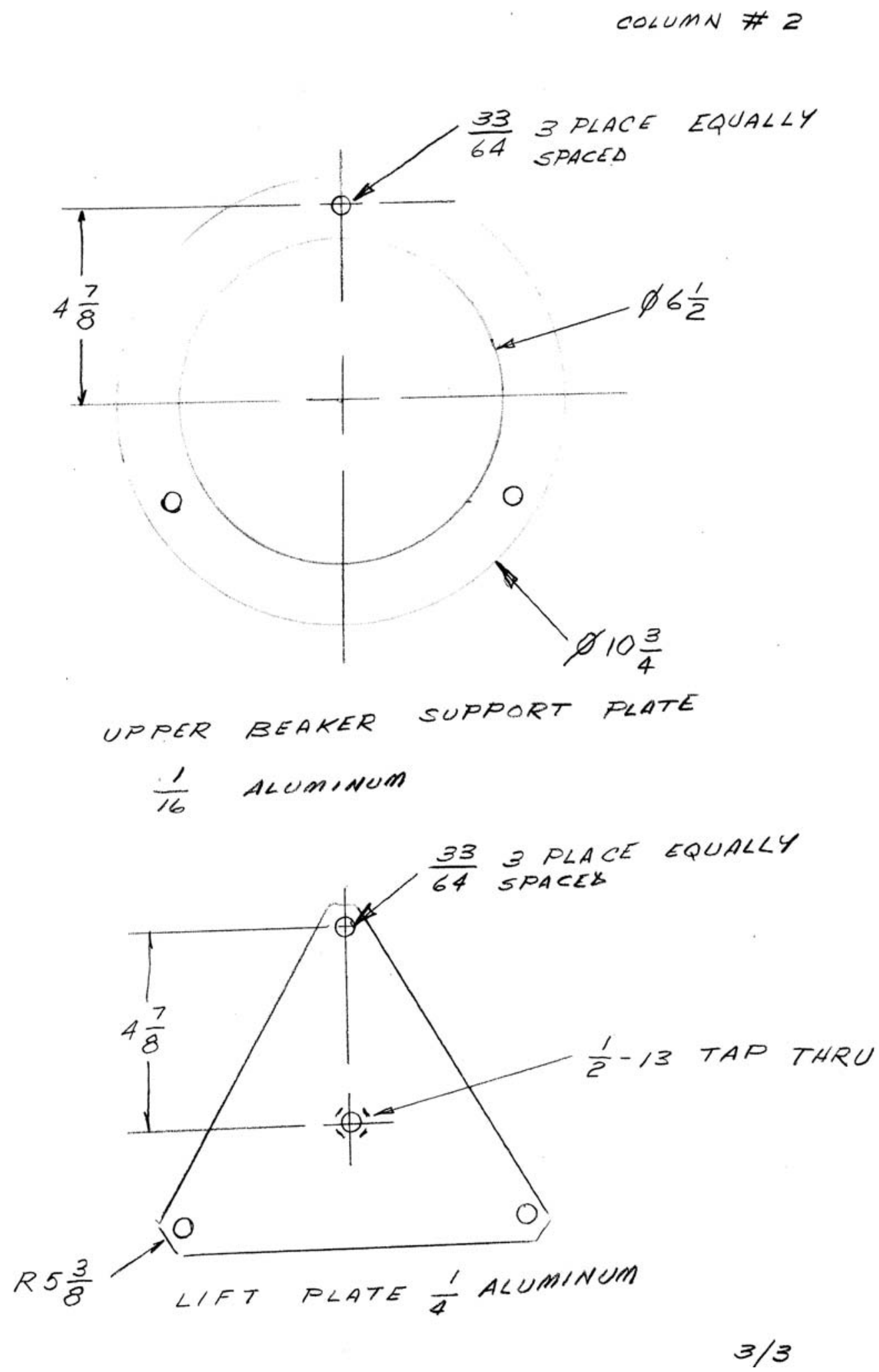



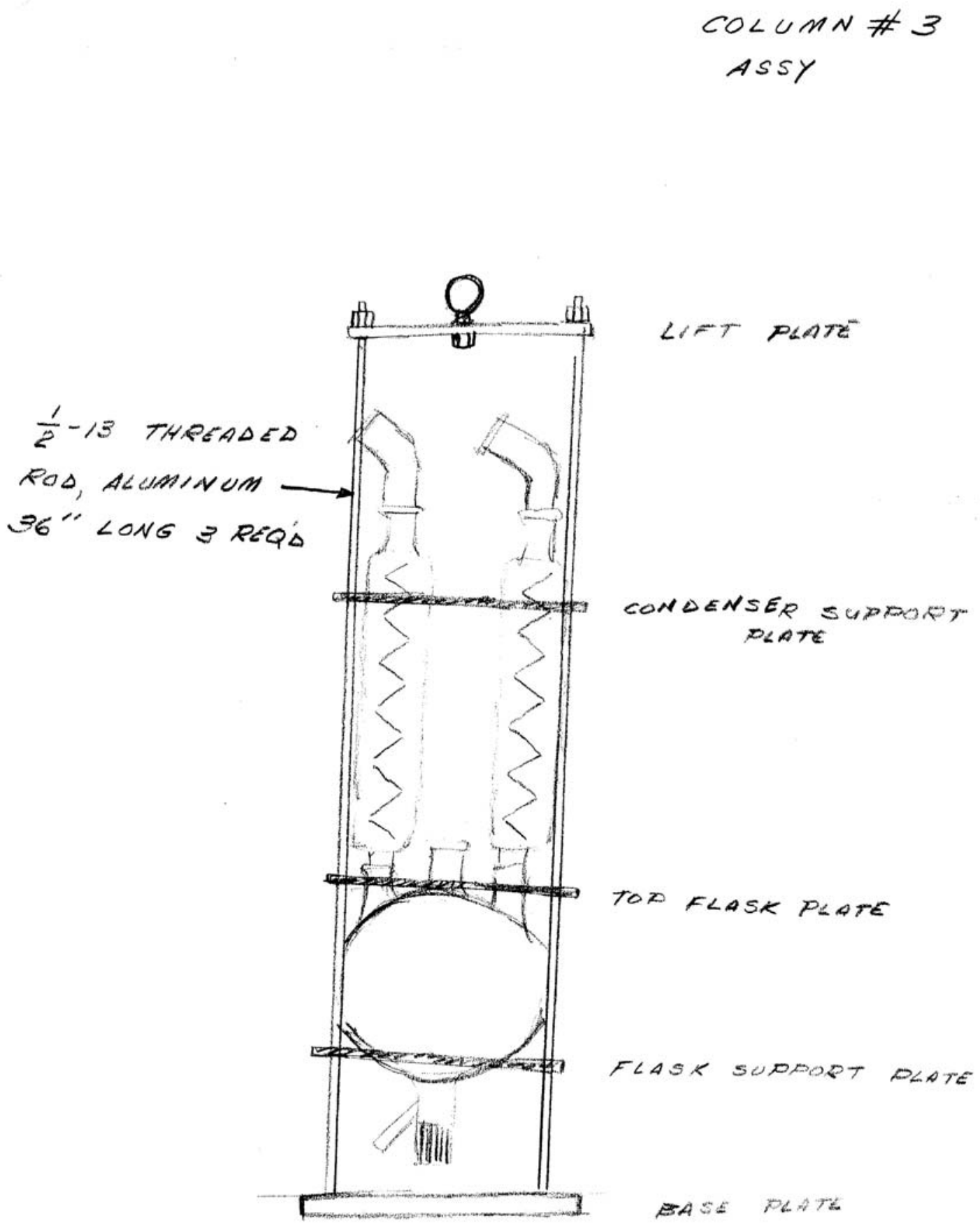

$1 / 4$ 

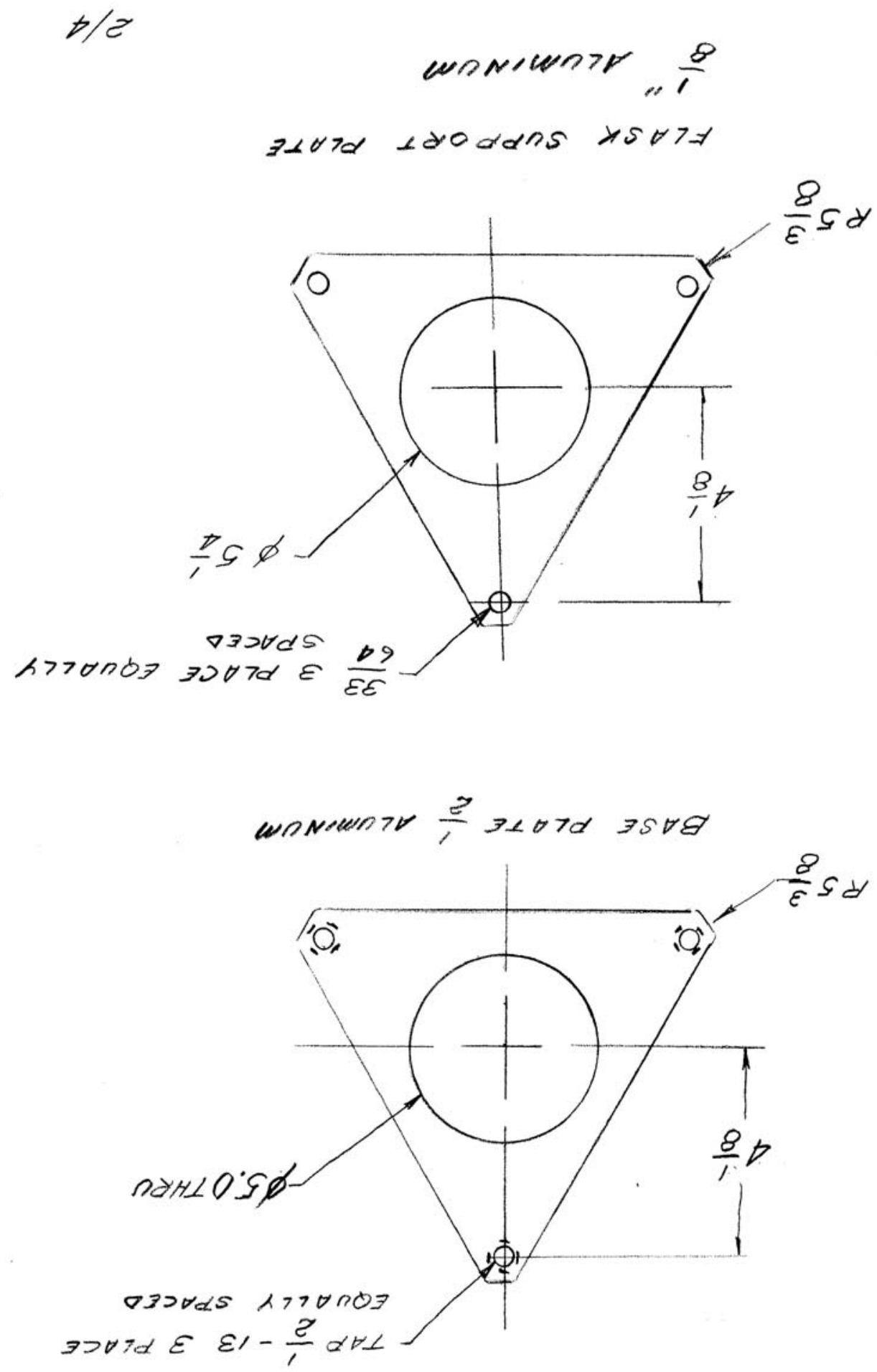

c 


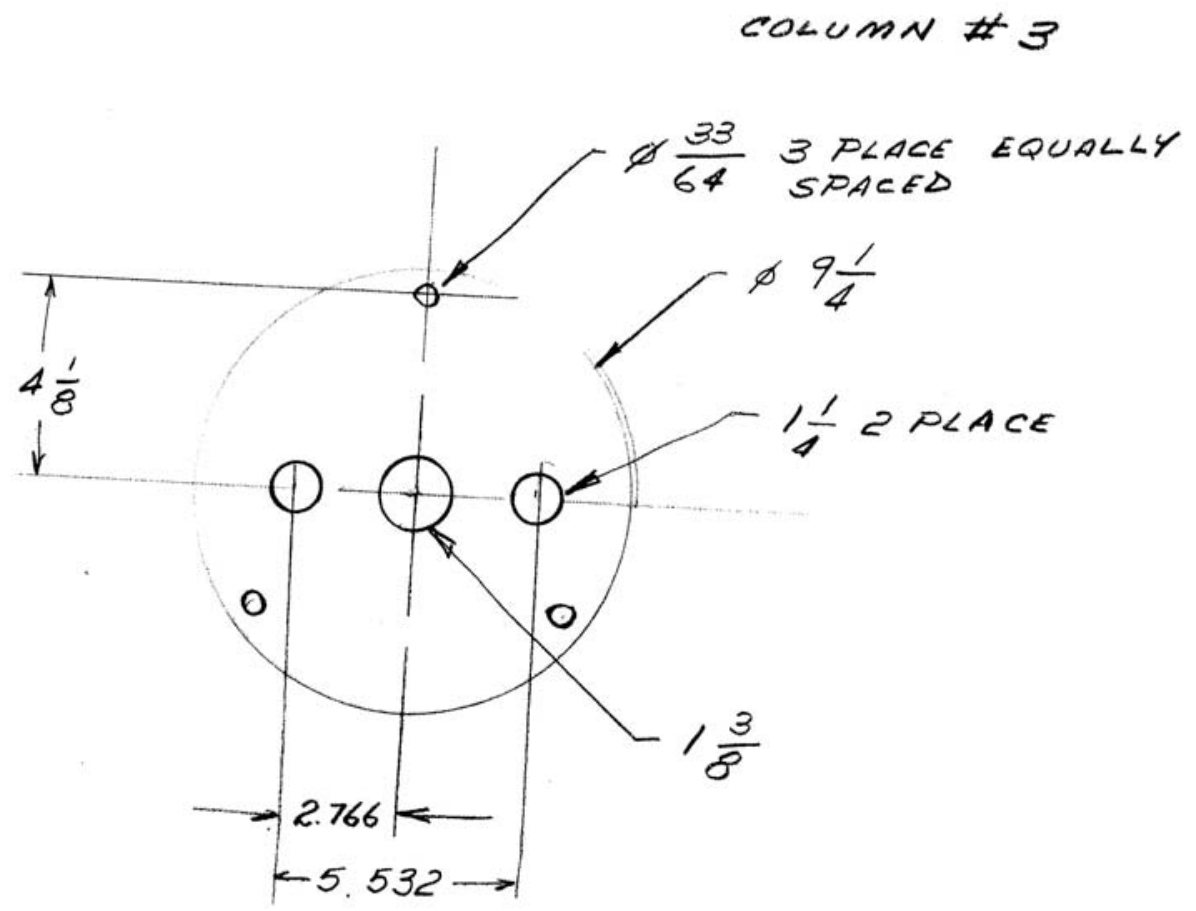

TOP OF FLASK PLATE $\frac{1}{16} \quad A \angle U M I N U M$

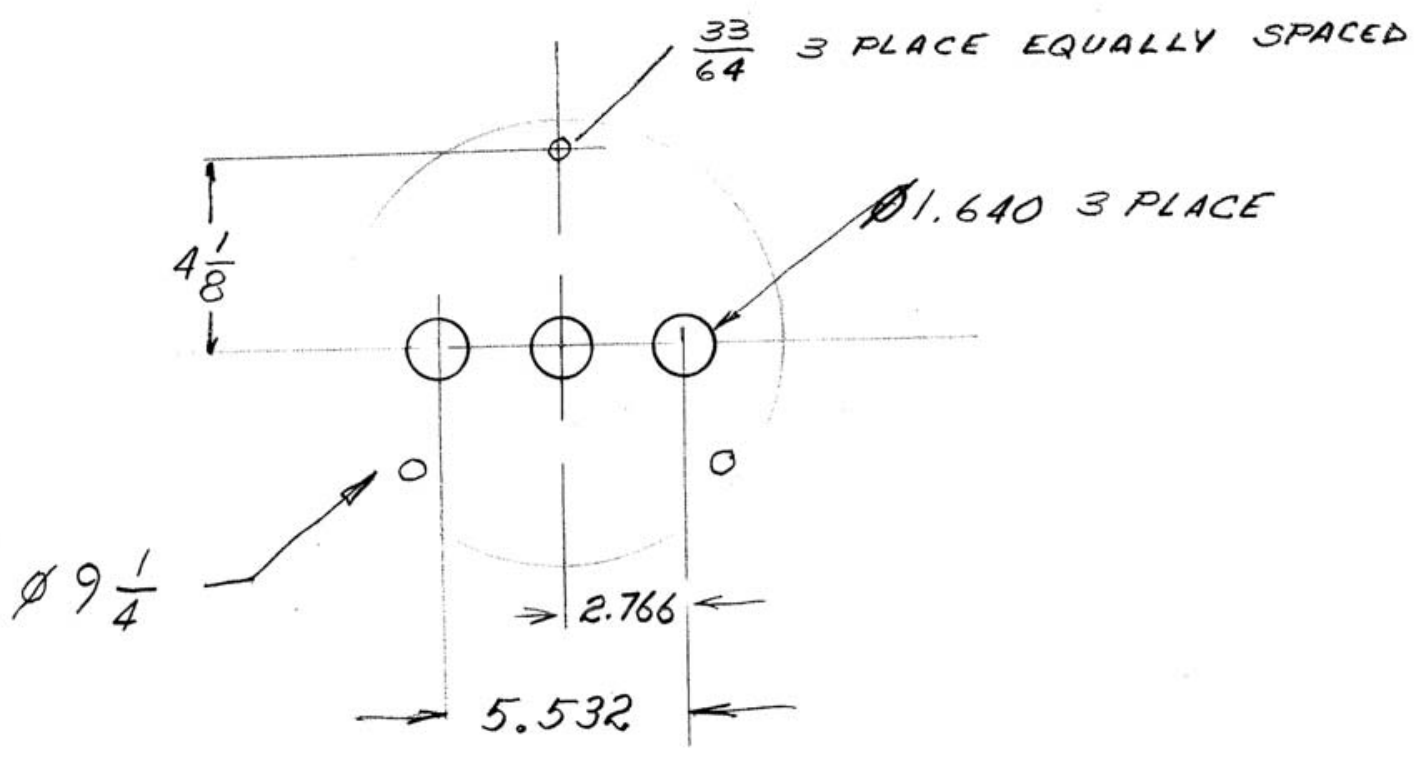
CONDENSEP SUPPOPT PLATE 


\section{coLUMN \#3}

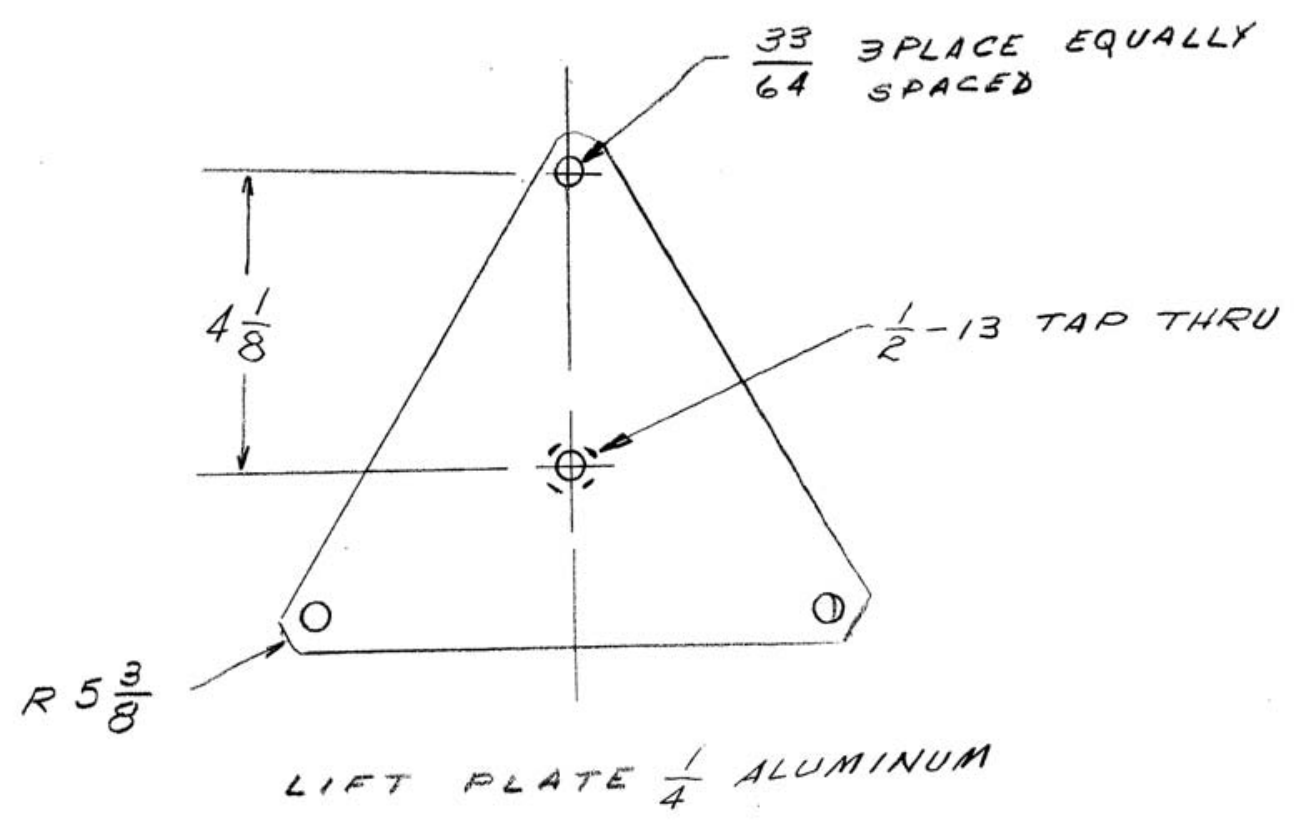

$4 / 4$ 


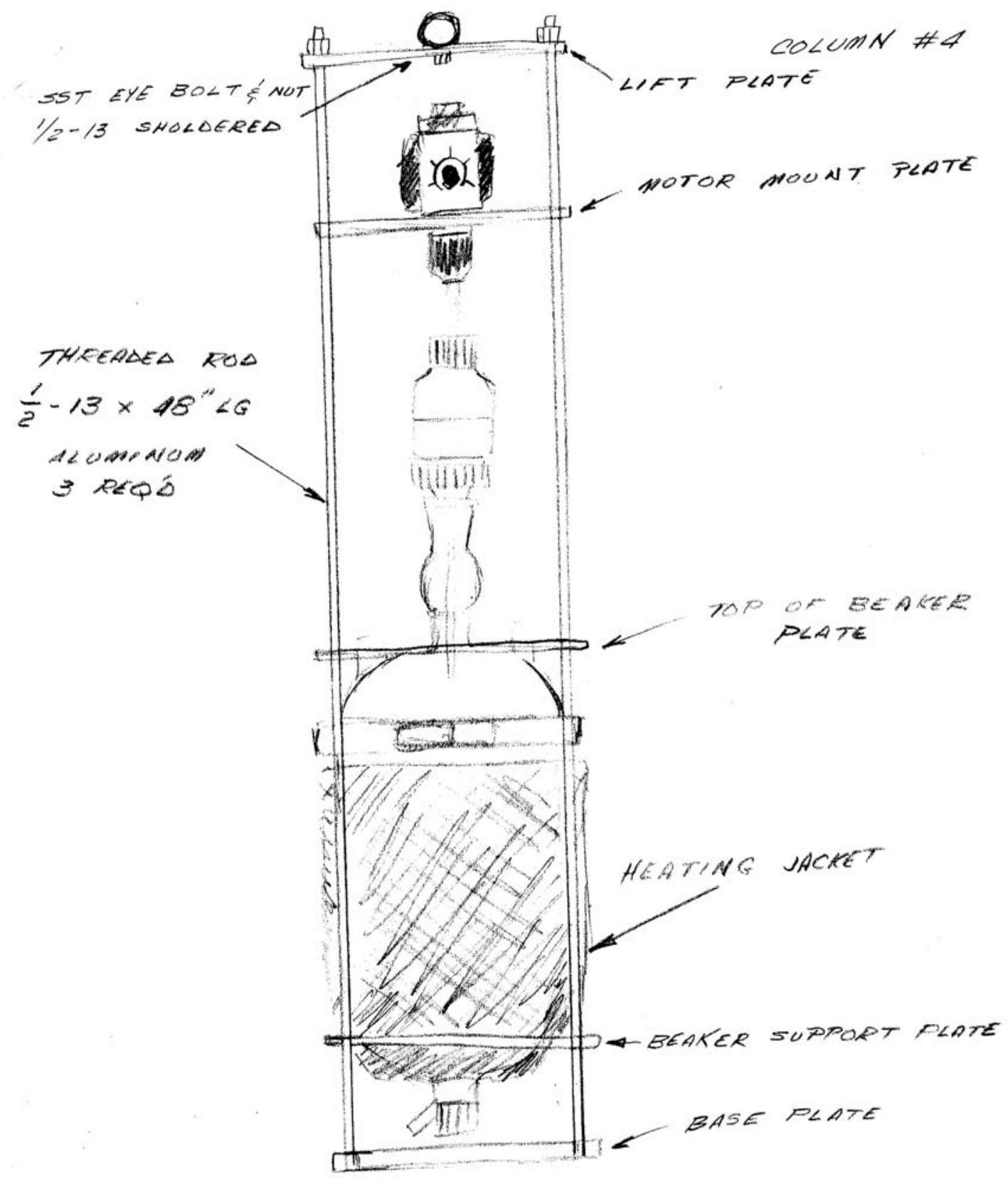

ASSY COLUMN \# 4

WEISHT 36 F 


$$
\begin{aligned}
& \text { COLUMN \# } 4 \\
& \text { TOTAL WEISAT } 36 \text { \# }
\end{aligned}
$$
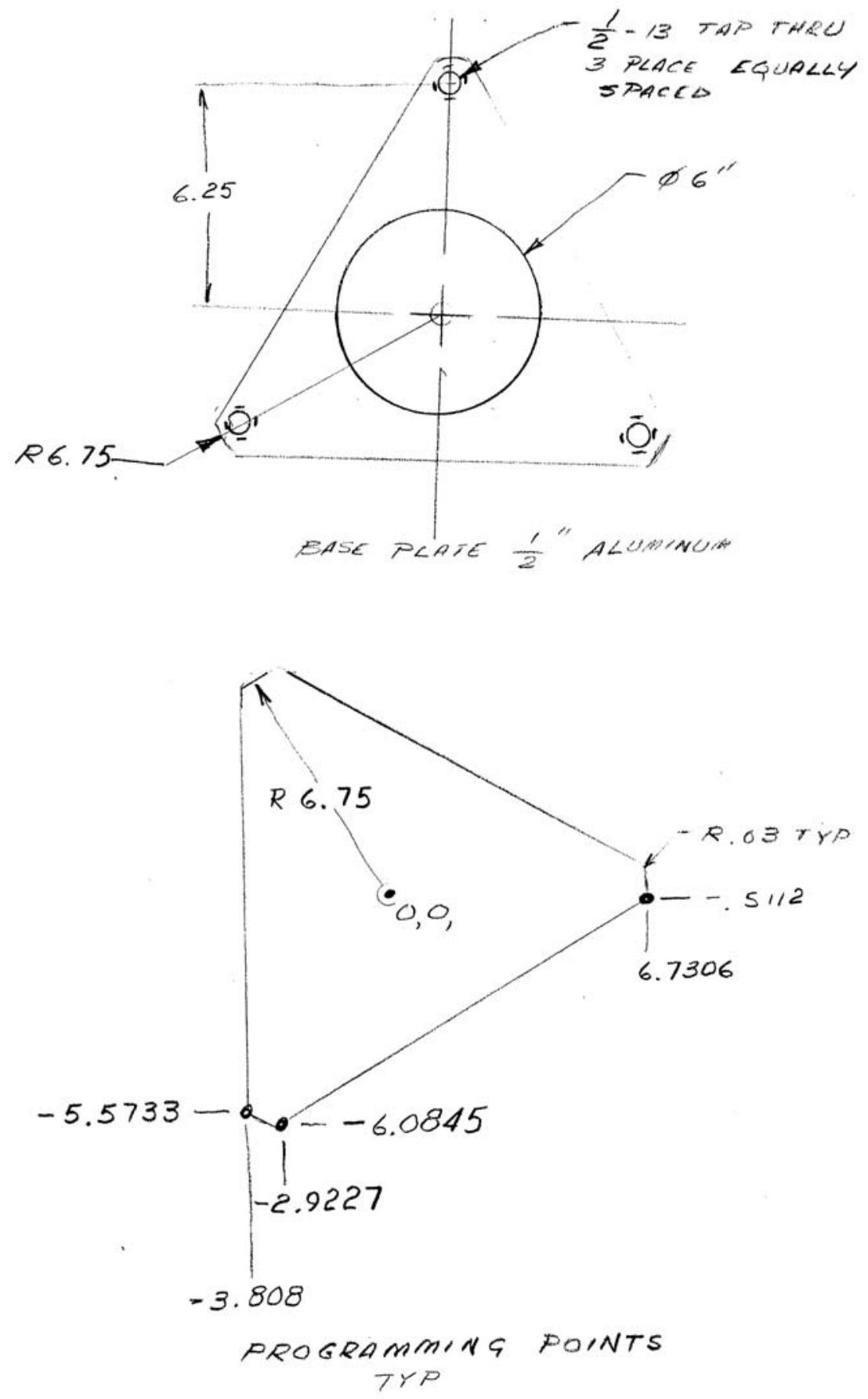


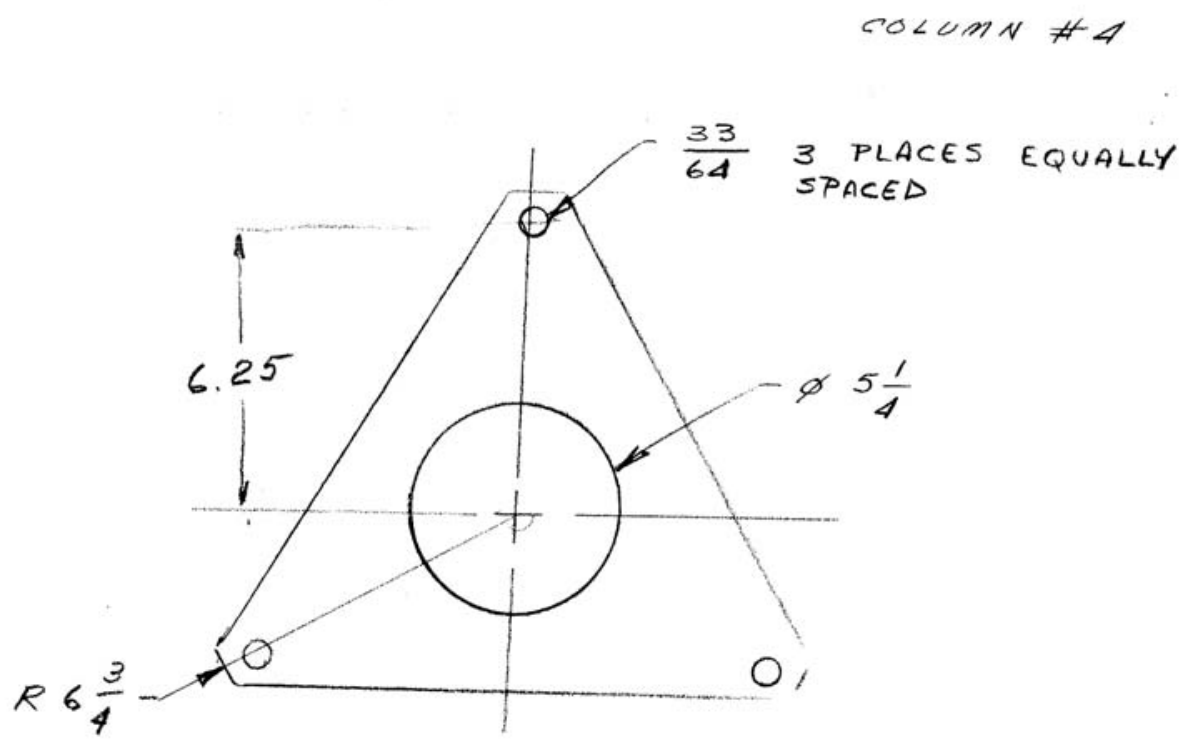

BEAKER SUPPORT PLATE, $\frac{1}{\sigma}$ ALUMINUM

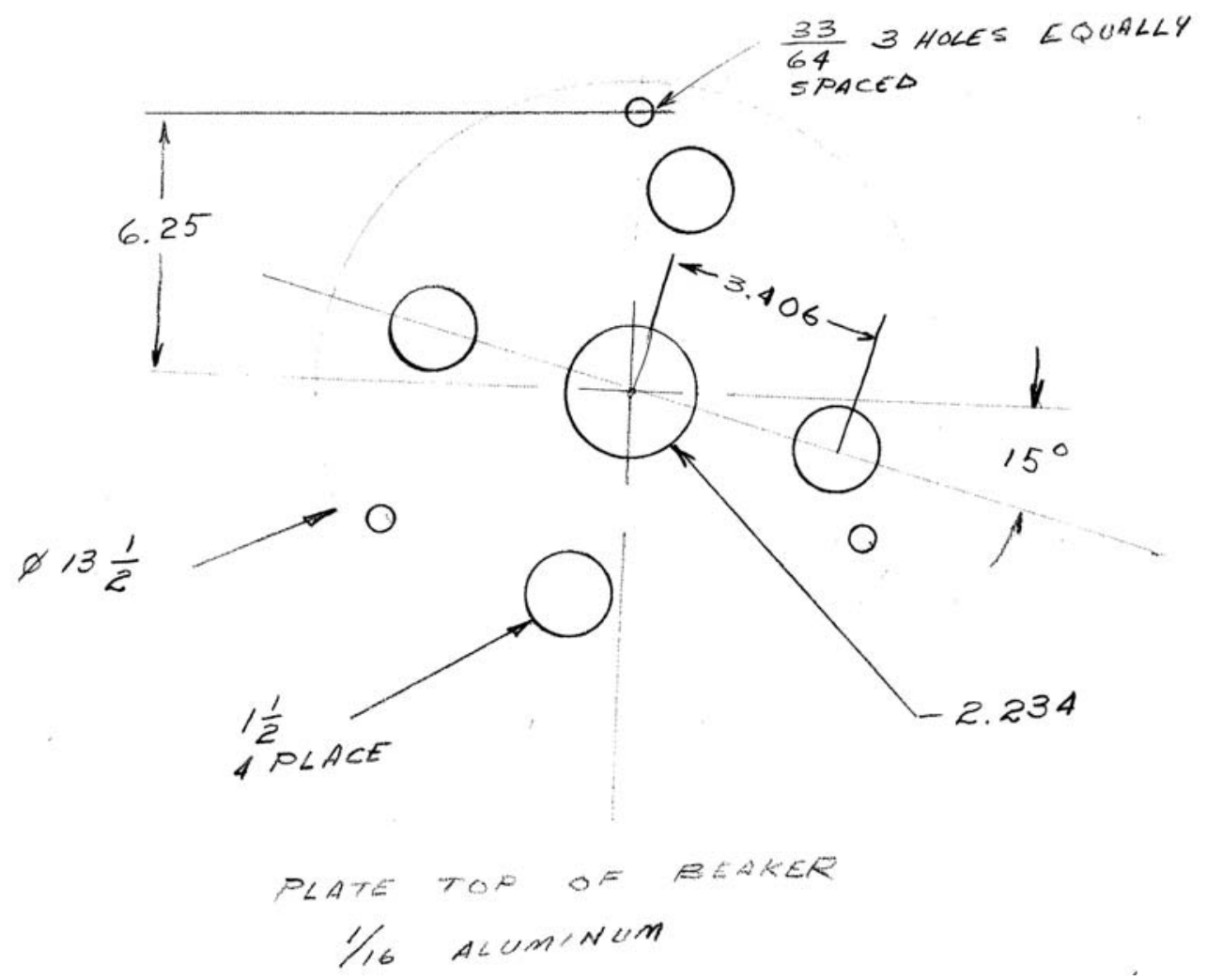




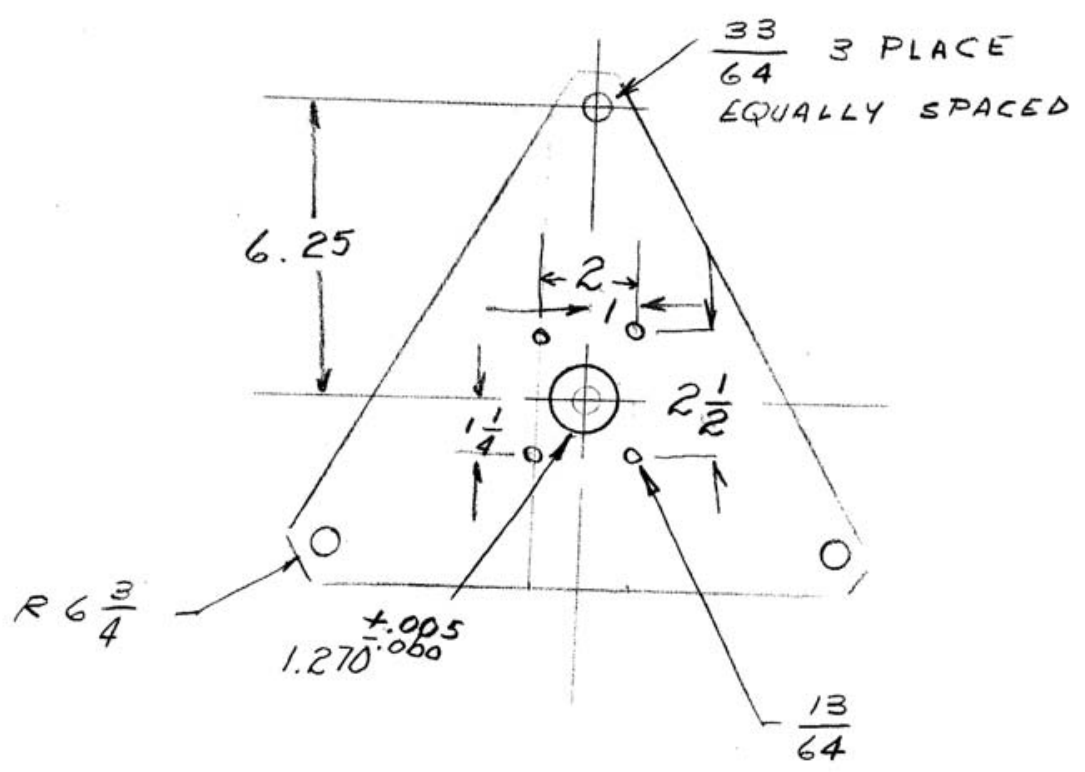

Motop no INT $\frac{1}{4}$ "ALUMINUM

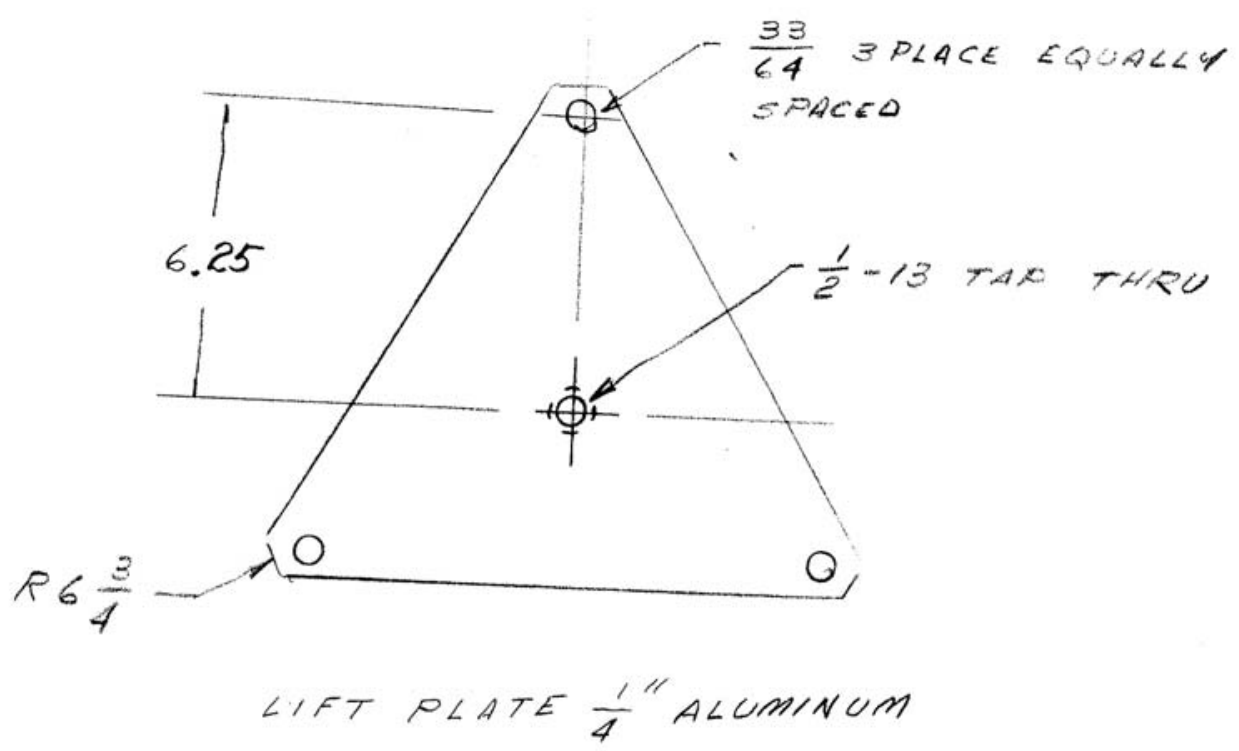


This page intentionally blank. 



\section{Argonne}

Nuclear Engineering Division

Argonne National Laboratory

9700 South Cass Avenue, Bldg. 208

Argonne, IL 60439-4854

www.anl.gov 